\title{
Chemisches Recycling von gemischten Kunststoffabfällen als ergänzender Recyclingpfad zur Erhöhung der Recyclingquote
}

\author{
A. Lechleitner - D. Schwabl - T. Schubert $(D)$ M. Bauer $\cdot$ M. Lehner
}

Online publiziert: 6. November 2019

(C) Der/die Autor(en) 2019

Zusammenfassung Kunststoffabfälle, speziell Verpackungsabfälle, liegen oft als Gemische mit hohem Verschmutzungsgrad vor. Die werkstoffliche Verwertung wird dadurch enorm erschwert, da die Sortierung und Reinigung dieser Fraktionen in vielen Fällen nicht ökonomisch sinnvoll oder technisch umsetzbar sind. Um diese Materialströme dennoch rohstofflich rezyklieren zu können, bietet das chemische Recycling eine vielversprechende Methode durch die Rückgewinnung von Einsatzstoffen für die Kunst- und Kraftstoffproduktion sowie für die petrochemische Industrie. Durch das Einwirken von Wärme, Katalysatoren und Lösungsmitteln werden dabei die Polymerketten in kürzere Einheiten bis hin zu Monomeren aufgespalten. Die dabei gewonnenen Kohlenwasserstoffe können dem Stoffkreislauf erneut zugeführt werden, um primäre Ressourcen zu ersetzen. Diese Technologien weisen eine hohe Toleranz gegenüber Störstoffen und Sortenunreinheiten auf und sind deshalb besonders attraktive Optionen für die Verwertung von verunreinigten Verpackungsabfällen. In den letzten 40 Jahren wurden hierzu verschiedene Ansätze zur Solvolyse und Pyrolyse mit und ohne Katalysator verfolgt, die zugrunde liegenden Mechanismen untersucht sowie zahlreiche Reaktorsysteme und Prozesswege entwickelt. Ein Überblick über die chemischen

Die AutorInnen A. Lechleitner,

D. Schwabl und T. Schubert trugen gleichermaßen zu dieser Arbeit bei und sind daher als Erstautoren anzusehen.

A. Lechleitner · T. Schubert $(\bowtie)$.

M. Bauer · M. Lehner

Verfahrenstechnik des industriellen Umweltschutzes,

Montanuniversität Leoben, Franz-Josef-Straße 18, 8700 Leoben, Österreich

teresa.schubert@unileoben.ac.at
Grundlagen, die entwickelten Verfahren und deren Werdegang gibt Aufschluss über die Chancen und Problematiken des Feedstockrecyclings als ergänzende Maßnahme zum werkstofflichen Recycling. Weiters werden die neuesten Forschungs- und Entwicklungsaktivitäten dargestellt, um den heutigen Entwicklungsstand und zukünftige Trends abzubilden und aufzuzeigen, dass das chemische Recycling eine potente Option zur Rückgewinnung von Rohstoffen und Schonung von Ressourcen darstellt.

Schlüsselwörter Chemisches Recycling · Feedstockrecycling • Kunststoffabfälle

Chemical recycling of mixed waste plastics as complementary process pathway to increase recycling rates

Abstract Waste plastics, especially packaging waste, arise as mixed fractions with high amounts of contaminants and impurities. To recycle these materials mechanically, costly sorting and purification is necessary, which is not always technical and economical feasible. Chemical or feedstock recycling provides a promising opportunity to recover these materials anyhow to obtain feedstock for the petrochemical industry, which can be processed to plastics again as well as to chemical commodities and fuels. To degrade the polymeric structure of the waste plastics to shorter hydrocarbons up to monomeric units, heat, catalysts and solvents can be applied. Depending on the specific technology, chemical recycling approaches have much higher tolerances towards mixed fractions and impurities and are capable to deal with contaminated and mixed packaging wastes. In the last 40 years, a broad range of technologies and processes were developed to realize chemical recycling for waste plastics. These technologies receive increased academic and industrial interest recently, due to increasing legal recycling rates and common awareness of plastics recycling. This review gives a comprehensive overview of the chemical fundamentals, developed processes and types of approaches as well as their opportunities and constraints. Furthermore, current developments in research and industry are summarized to point out recent activities in feedstock recycling as promising complementary option for waste plastics recycling.

Keywords Chemical recycling . Feedstock recycling - Waste plastics

\section{Kunststoffrecycling}

Die weltweite Kunststoffproduktion ist laut PlasticsEurope (PlasticsEurope AISBL 2018) im Jahr 2017 auf fast 350 Mio. Tonnen angewachsen, davon entfallen knapp 65Mio. Tonnen allein auf die 28 EU-Staaten sowie die Schweiz und Norwegen (in weiterer Folge vereinfacht als Europa bezeichnet). Immerhin 50 Mio. Tonnen (PlasticsEurope AISBL 2018) davon werden auch in diesen Staaten verbraucht, wobei die Hälfte hiervon auf nur drei Kunststoffsorten (Polyethylen, Polypropylen und Polystyrol) der Gruppe der sogenannten Polyolefine entfällt. Diese Gruppe besitzt somit eine überproportionale Bedeutung, wenn man die nahezu endlose Vielfalt an Kunststoffen betrachtet. Ihre Relevanz für die Abfallwirtschaft wird umso größer, wenn man bedenkt, dass Polyolefine den Hauptwerkstoff für Kunststoffverpackungen und -behältnisse darstellen, welche im Vergleich zu anderen Polymerprodukten eine sehr kurze Lebensdauer aufweisen. Diese Polymergruppe ist daher in den jährlich etwa 27 Mio. Tonnen in Europa anfallender Kunststoffabfälle überproportional vertreten. 


\begin{tabular}{l|l|l|l|l|l}
\hline \multicolumn{7}{l}{ Tab. 1 Heizwerte verschiedener Energieträger bei 25 in MJ/kg nach. (Nach Martens and Goldmann 2016) } \\
\hline Hausmüll & $2,5-12,0$ & Kohle & $25,0-35,0$ & Polypropylen & 44,0 \\
\hline Polyethylenterephthalat (PET) & 4,2 & Polyamid (PA) & 31,0 & Polyethylen & 46,1 \\
\hline Papier & 17,0 & Erdgas & $32,0-45,0$ & Propan & 46,3 \\
\hline Holz & 18,0 & Polystyrol & 40,2 & Methan & 50,0 \\
\hline Polyvinylchlorid (PVC) & 18,0 & Erdöl & 42,8 & Wasserstoff & 120,0 \\
\hline
\end{tabular}

Die ökologisch und wirtschaftlich sinnvolle Behandlung von Kunststoffabfällen stellt technisch eine der Herausforderungen der modernen Abfallwirtschaft dar. Ebenjene Eigenschaften, welche den Siegeszug der Kunststoffe als Produkte in fast alle Lebensbereiche ermöglich haben (allen voran die hohe Beständigkeit, aber auch die gute Veränderbarkeit physikalischer Eigenschaften durch Zusätze und kostengünstige Herstellung), machen Kunststoff als Abfall zu einer Umweltgefahr und einem anspruchsvollen Reststoff.

Es haben sich drei Behandlungsstrategien etabliert, Kunststoffabfälle nach Gebrauchsende zu verbringen:

- Die Deponierung hat Massen- und Volumsreduktion und sichere Lagerung von Abfällen in einem Endlager zum Ziel. Sie stellt eine simple Beseitigungsform ohne Verwertungspotenzial dar, die in einigen Staaten Europas wie etwa Österreich und Deutschland für Kunststoffe aufgrund des hohen Anteils an organischem Kohlenstoff verboten ist.

- Das thermische oder auch energetische Recycling nützt den Energieinhalt von Abfällen und kann damit zur Einsparung von primären Energieträgern beitragen. Für Kunststoffe ist dieser Verwertungsweg interessant, da sie als Erdölprodukte teilweise ähnlich hohe Heizwerte wie fossile Energieträger ausweisen, wie Tab. 1 zeigt.

- Beim Recycling zuletzt werden Werkstoffe oder deren chemische Bausteine aus Abfällen rückgewonnen, um daraus erneut Kunststofferzeugnisse zu produzieren und so primäre Rohstoffe einzusparen. Zum Beispiel das österreichische Abfallwirtschaftsgesetz definiert hierbei stoffliches Recycling so, dass alle Verwertungsmaßnahmen darunter fallen, die eine Substitution von Primärrohstoffen bewirken und die nicht unter thermische Verwertung fallen (Bundesgesetz über eine nachhaltige Abfallwirtschaft (Abfallwirtschaftsgesetz 2002 - AWG 2002)). Dabei kann weiters zwischen mechanischem oder werkstofflichem und chemischem oder rohstofflichem Recycling unterschieden werden, wobei beim ersteren die Polymerstruktur nahezu unverändert durch erneutes Aufschmelzen in Sekundärprodukte überführt wird. Das chemische Recycling auf der anderen Seite basiert auf der Rezyklierung chemischer Bausteine, die durch Kettenspaltung gewonnen und in folgenden Verarbeitungsschritten wieder chemisch repolymerisiert werden.

In den vergangenen Jahrzehnten haben sich aus diesen drei Verwertungswegen in den einzelnen Staaten unterschiedliche Abfallwirtschaftssysteme entwickelt und auf die spezifischen Bedürfnisse des Landes, politische sowie wirtschaftliche Rahmenbedingungen eingestellt. So verwundert es nicht, dass deren Darstellung wie in Abb. $1 \mathrm{zu}$ sehen, eine unterschiedliche Ausrichtung offenbart. Staaten mit höher entwickelten Abfallwirtschaftssystemen setzen auf eine Kombination aus thermischem und stofflichem Recycling und können so den Abfall zu einem gewissen $\mathrm{Ma}$ zur Gewinnung von Sekundärrohstoffen und -energieträgern nutzen. Auf der anderen Seite sind Staaten, deren Abfallwirtschaftssysteme sich im Aufbau befinden, oft noch zu einem hohen Maß auf die Deponierung angewiesen, wobei andere Verwertungswege nur $\mathrm{zu}$ einem kleinen Teil realisiert sind. Um eine weitere Entwicklung der Abfallwirtschaftssysteme anzuregen, hat die Europäische Kommission im Juli 2018 die überarbeitete Richtlinie über Verpackungen und Verpackungsabfälle erlassen, worin für 2025 und 2030 Recyclingziele für Kunststoffverpackungen von 50 bzw. $55 \%$ gefordert sind (Europäische Kommission 2018).

\section{Trenntechnik Kunststoffabfälle}

Eine Kernaufgabe bei der Erreichung dieser Ziele wird den Betrieben der Abfallaufbereitung zukommen, denn die bisher vergleichsweise geringen Mengen des stofflichen Recyclings hängen vor allem damit zusammen, dass beim mechanischen Recycling die Anforde- rungen, die an die Reinheit des Recyclingkunststoffs gestellt werden, im Bereich jenseits $99 \%$ liegen, damit die daraus erstellten Produkte mit Primärprodukten konkurrieren können. Die Notwendigkeit dieser geringen Fremdstoffanteile lässt sich an Abb. 2 erkennen. Beim werkstofflichen Recycling werden Altkunststoffe, gleich wie bei der Verarbeitung primärer Produkte, mittels Extrudern erhitzt und so formbar gemacht, um sie verarbeiten $\mathrm{zu}$ können. Verschiedene Kunststoffsorten weisen aber ein unterschiedliches Erwärmungsverhalten auf und erweichen in nicht sortenreinen Mischungen zu unterschiedlichen Verarbeitungszeitpunkten. Dies kann zu Schäden am Extruder und Einschlüssen im erzeugten Werkstoff führen. Solche Einschlüsse fremder Polymere wirken in einem Kunststoff wie ein Fehler im Kristallgitter eines Minerals und es entstehen bei Belastung Risse, welche zu verfrühtem Versagen des Werkstoffs führen können. Schon geringe Verunreinigungen können somit die physikalischen Eigenschaften eines Polymerwerkstoffs dramatisch verändern.

Um entsprechende reine Kunststoffprodukte aus Abfällen abzutrennen, stehen die Abfallaufbereiter vor der Aufgabe, teilweise sehr heterogene Inputströme bearbeiten zu müssen. Häufige Verunreinigungen wie Metalle und Glas können meist einfach durch Windsichtung, Magnet- und Wirbelstromscheidung entfernt werden, wodurch als qualitätsentscheidender Schritt meist die Trennung der verschiedenen Kunststoffsorten untereinander bleibt.

In diesem Bereich haben sich zwei Technologien hervorgetan, mit denen dieser Aufgabe begegnet werden kann. Zum einen ist es die Dichtetrennung, welche mit Flüssigkeiten verschiedener Dichte durchgeführt wird und sich des Umstands bedient, dass sich - wie in Tab. 2 ersichtlich - verschiedene Kunststoffe durch unterschiedliche Dichten auszeichnen. Je nach konkreter Trennaufgabe können weitverbreitete Schwimm-Sink-Tanks, wie sie etwa im Flaschenrecycling, Hydrozyklone oder Sortierzentrifugen eingesetzt werden. 


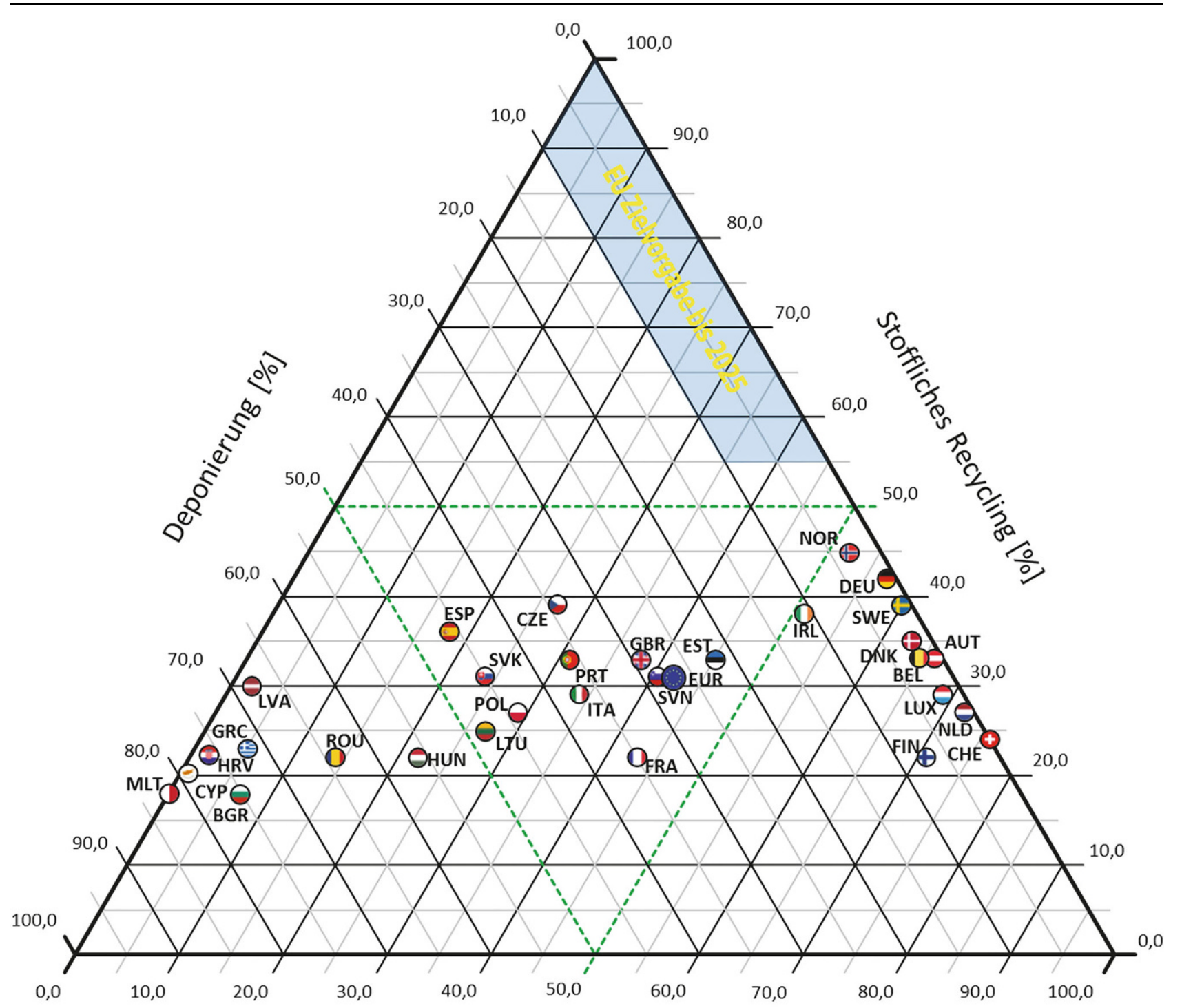

Thermisches Recycling [\%]

Abb. 1 Verwertungswege für Kunststoffe in den Ländern Europas 2018. (Nach Europäische Kommission 2018; PlasticsEurope AISBL2018)

Die andere bekannte Technologie ist die optische Sortierung, ein trockener Prozess, welcher unter den Oberbegriff der Klaube-Trenntechnologien fällt. Hierbei werden die einzelnen Partikel, welche sich über ein Förderband bewegen von Sensoren erfasst, die ihre Position und spektrale Eigenschaften feststellen. Über das gemessene Spektrum können je nach Vorgabe Eigenschaften wie Farbe, Form, Materialzusammensetzung, Feuchtigkeit u.v.m. festgestellt werden. Es wird dann automatisch entschieden, ob diese Partikel den gewünschten Eigenschaften entsprechen oder nicht und dann entsprechend mittels Luftdruckdüse, Klappe oder Ähnlichem in den korrekten Materialaustrag überführt. Hatten diese Apparate früher wegen unzureichender Rechenleistungen nur geringe Durchsätze, so können nun bereits bis zu 10 Tonnen pro Stunde und Meter Förderbandbreite erreicht werden (Martens and Goldmann 2016).

Eine weniger verbreitete Technologie, die hier dennoch genannt werden soll, ist die elektrostatische Trennung mittels triboelektrischer Freifallscheidung. Hierbei wird ausgenutzt, dass Kunststoffpartikel unterschiedlicher chemischer Zusammensetzung in einem Reaktionsraum bei intensivem Kontakt untereinander sowie der Apparatewandung unterschiedliche Oberflächenladungen erhalten. Diese Oberflächenladungen können die Partikel infolge ihrer geringen Leitfähigkeit nur sehr langsam wieder abgeben, wodurch sie anschließend im elektrischen Feld eines Freifallscheiders abgetrennt werden können. Ob sich Kunststoffe im Kontakt miteinander positiv oder negativ laden, kann der sogenannten triboelektrischen Spannungsreihe entnommen werden.

Rein technisch gesehen ist es daher möglich, auch aus einem vergleichsweise heterogenen Einsatzstrom hochreine Kunststoffprodukte abzutrennen. Jedoch bedarf es dabei einer Kombina- 

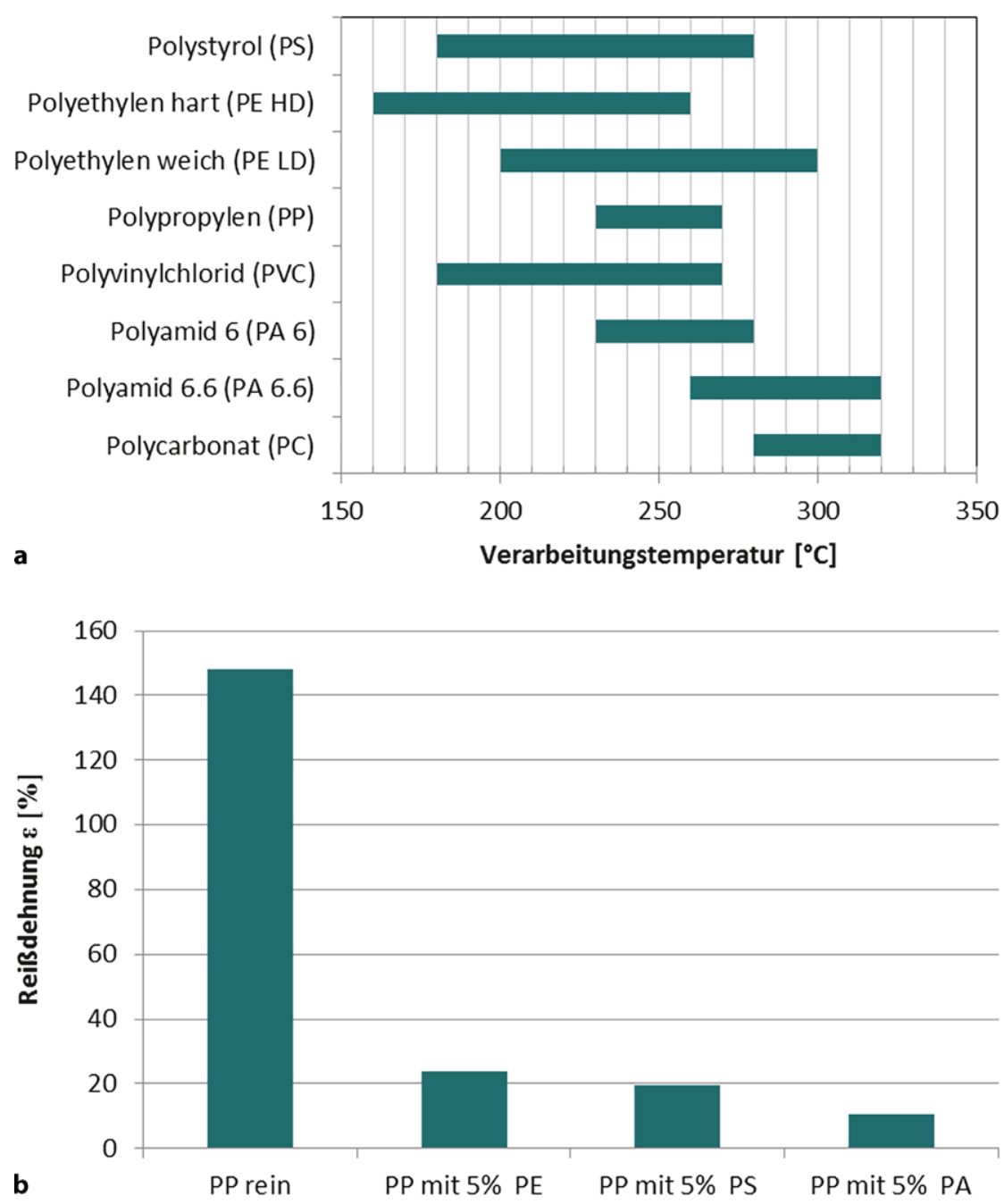

Abb. 2 Verarbeitungstemperatur unterschiedlicher Kunststoffe (a); Änderung der Reißdehnung von Polypropylen durch Verunreinigungen (b). (Nach Michaeli et al. 1993)

tion mehrerer Prozessschritte, da diese entweder auf eine möglichst vollständige Abtrennung der Verunreinigung, wodurch Verluste an Wertstoffen in Kauf genommen werden müssen, oder möglichst vollständige Rückgewinnung der Wertstoffe, wodurch es zu Produktverunreinigungen kommt, ausgelegt werden können. Je höher die Steigerung des Wertstoffinhalts zwischen Einsatzstoff und Produkt sein soll, umso mehr immer feiner arbeitende Prozessschritte sind daher erforderlich, wodurch es zu einem exponentiellen Wachstum der Aufbereitungskosten kommt.

Der Verkaufspreis des schlussendlich hergestellten Produkts muss diese und andere Kosten tragen und gleichzeitig mit jenem von primären Kunststoffprodukten konkurrieren können, damit ein mechanisches Recycling wirtschaftlich ist. Dies ist aufgrund der geringen
Somit lassen sich Monomere, petrochemische Grundstoffe und Synthesegas aus Abfallströmen gewinnen, wobei die Einteilung je nach einwirkender Triebkraft zur Kettenspaltung erfolgt (siehe Abb. 3). Man unterscheidet hierbei zwischen

- Hydrocracking,

- thermokatalytischer Konversion oder katalytischem Cracken,

- thermischer Konversion oder Pyrolyse,

- chemischer Depolymerisation oder Solvolyse und

- Gasifizierung oder Vergasung,

die jeweils absteigende Ansprüche an den Reinheitsgrad der Einsatzfraktionen stellen. Im folgenden Kapitel wird auf die unterschiedlichen Ansätze, ihre Charakteristika und ihre Vor- und Nachteile eingegangen.

\subsection{Chemische Depolymerisation - Solvolyse}

Bei der chemischen Depolymerisation, die auch Solvo- oder Chemolyse genannt wird, werden die Polymere des Kunststoffabfalls mithilfe von chemischen Reagenzien in ihre Monomerbestandteile zerteilt, welche entweder zur erneuten Erzeugung desselben Werkstoffs oder als Ausgangsstoff für andere chemische Synthesen dienen können. Chemisch gesehen läuft dabei die Umkehrreaktion der Polykondensation oder eine artverwandte Reaktion dieser durch Einbau von kleinen Molekülen ab. Daraus folgt, dass diese Technologie ausschließlich für Polykondensationskunststoffe wie Polyester und Polyamide Anwendung finden kann, da diese durch Abspaltung von Wasser oder anderen Verbindungen synthetisiert werden. Der Hauptteil der Kunststoffabfälle besteht jedoch aus Polyolefinen, welche sich nicht zur Chemolyse eignen und somit mit solch einer Technologie nicht dem wertstofflichen Recycling zugeführt werden können (Clarke et al. 1999; Ragaert et al. 2017).

Für die Solvolyse kommen unterschiedliche Lösungsmittel zum Einsatz, wodurch dieser Ansatz des tertiären Recyclings weiter unterteilt werden kann in die vorherrschenden Wege der

- Glykolyse,

- Methanolyse,

- Hydrolyse sowie

- Ammonolyse und Aminolyse. 
Tab. 2 Dichtespektren verschiedener Kunststoffe in $\mathrm{g} / \mathrm{cm}^{3}$. (Nach Bilitewski et al. 2000)

\begin{tabular}{|l|l|l|l}
\hline Polypropylen (PP) & $0,90-0,91$ & Polyamid 6 (PA 6) & 1,13 \\
\hline Polyethylen (PE) & $0,91-0,96$ & Polyethylenterephthalat (PET) & 1,37 \\
\hline Acrylnitril/Butadien/Styrol (ABS) & $1,04-1,06$ & Polyvinylchlorid (PVC) & $1,38-1,55$ \\
\hline Polystyrol (PS) & 1,05 & Polyoxymethylen (POM) & $1,41-1,42$
\end{tabular}

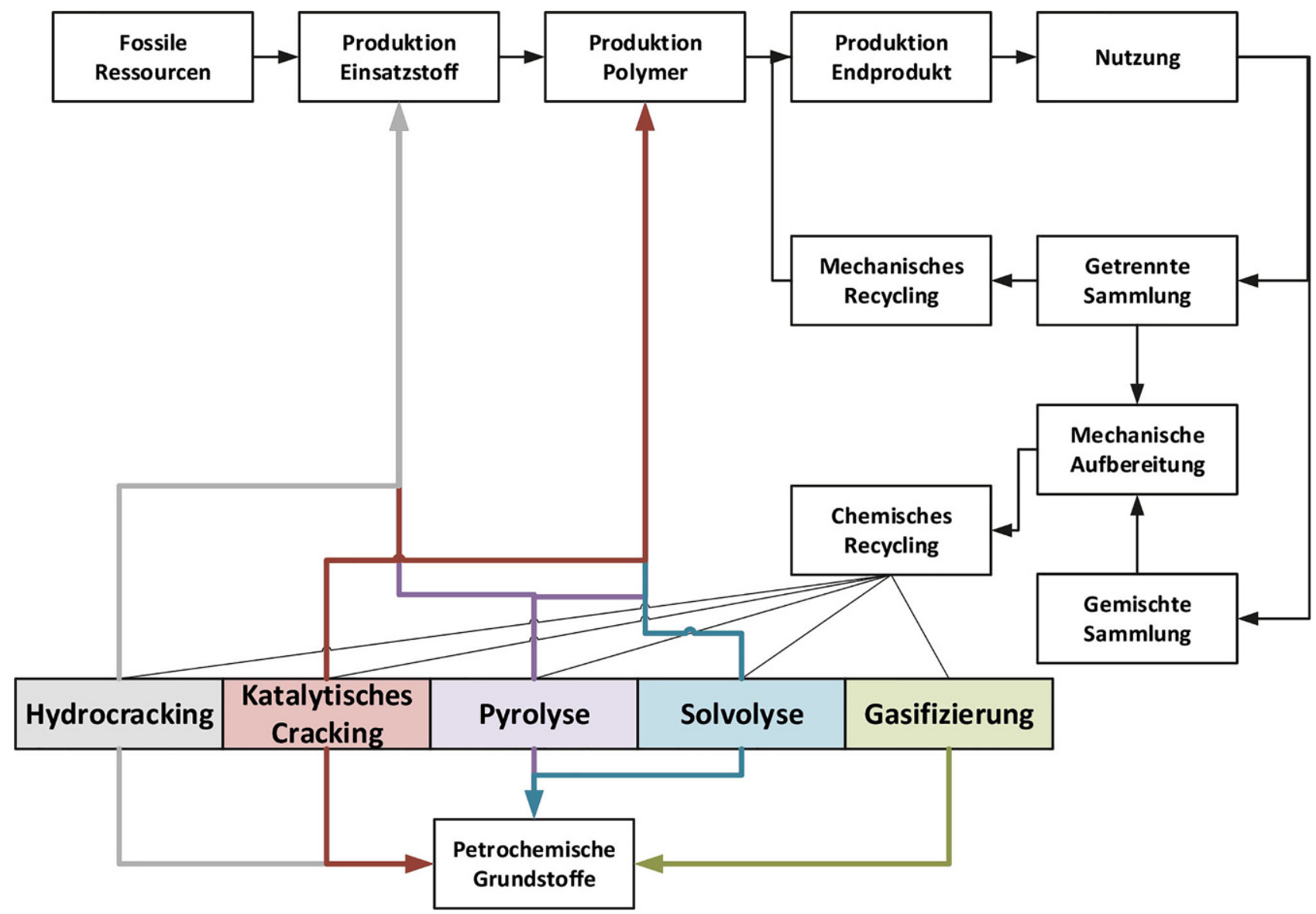

Abb. 3 Möglichkeiten des chemischen Recyclings im Lebenszyklus von Kunststoffprodukten

Der Einsatz unterschiedlicher Reagenzien führt jeweils $\mathrm{zu}$ verschiedenen Recyclingprodukten, wobei auch Kombinationen der gelisteten Strategien zur Anwendung kommen können. Am Beispiel von PET zeigt Abb. 4 die Vielzahl der Produktverbindungen und deren potenzielle Verwendung, die sich durch Reaktion mit den jeweiligen Lösungsmitteln ergeben.

Die Glykolyse von PET, die älteste und einfachste Chemolyse-Technologie, setzt unterschiedliche Glykole (Ethylenglykol, Diethylenglykol, Propylenglykol, Dipropylenglykol) zur Aufspaltung der Polymere bei Temperaturen zwischen 180 und $240^{\circ} \mathrm{C}$ ein. Dabei diffundiert das Glykol in die Struktur des Polymers, welche anschließend aufquillt und das Glykol mit der Esterbindung reagiert. Dieser Mechanis- mus wird durch eine große, spezifische Oberfläche, etwa bei stark zerkleinertem Einsatzmaterial, begünstigt und kann durch Katalysatoren wie Zeolithe, ionische Flüssigkeiten oder Metallverbindungen und Kombinationen mit anderen Lösungsmitteln beschleunigt und verbessert werden. Auch der Einsatz von Glyzerin ist möglich, wobei die weitverbreitetste Methode Ethylenglykol verwendet (Pardal and Tersac 2006). Die dabei entstehenden Verbindungen wie Bis(hydroxyethyl)terephthalat (BHET) und PET-Glykolysate können gereinigt sowohl zur Synthese von Polyurethanen, Copolyestern und ungesättigten Harzen oder auch erneut bei der Herstellung von PET eingesetzt werden (Sheel and Pant 2019).

Bei der Methanolyse, die bei ähnlichen Temperaturen $\left(180\right.$ bis $\left.280^{\circ} \mathrm{C}\right)$ aber auch höheren Drücken von 20 bis 40 bar abläuft, wird die Polyesterstruktur durch Methanol meist in Anwesenheit eines Katalysators wie Zink-, Magnesium- oder Cobaltacetat zur Unterstützung der Umesterung aufgespalten. Dabei werden gleichermaßen Rohstoffe zur erneuten PET-Synthese, in diesem Fall Dimethyltherephthalat und Ethylenglykol, rückgewonnen, wobei diese Grundstoffe eine höhere Reinheit als bei der Glykolyse aufweisen, allerdings nicht solch breite Anwendungsfelder bedienen können. Die hohen Drücke in der Reaktionszone erschweren jedoch im Vergleich die Einbringung der Einsatzstoffe bei kontinuierlicher Prozessführung, wodurch meist die Nachteile des Batchbetriebs bei der Methanolyse in Kauf genommen werden müssen. Weiters erfordert diese Technologie hö- 


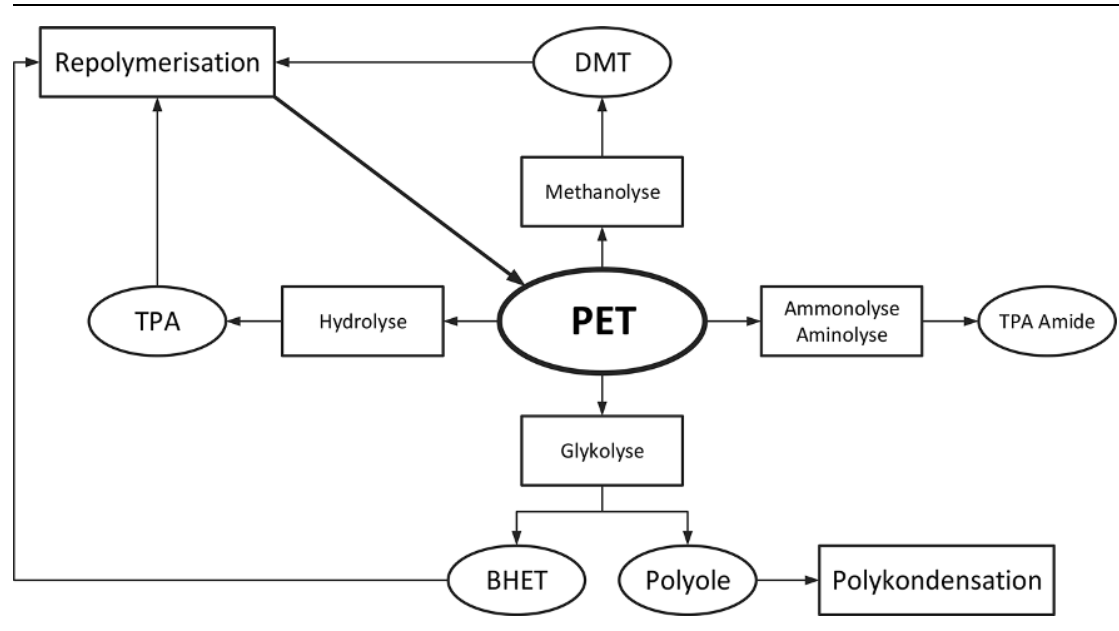

Abb. 4 Pfade der chemischen Depolymerisation von PET. (Nach Clarke et al. 1999)

here Reinheiten der Abfallfraktionen und die Investitionskosten fallen höher aus, da der Einsatz von überhitztem Methanol strenge Sicherheitsmaßnahmen fordert.

Durch Hydrolyse bei neutralen, sauren und basischen Bedingungen durch Zugabe von zum Beispiel Schwefelsäure oder Natriumhydroxid zu Wasser wird PET in Terephthalsäure (englisch terephthalic acid, TPA) und Ethylenglykol aufgespalten. Da die gewonnene TPA niedrige Reinheiten aufweist und weitere Reinigungsschritte für eine erneute Polykondensation erforderlich macht, sowie die Zersetzung vergleichsweise langsam vonstattengeht, kommt der Hydrolyse geringere Bedeutung zu, obwohl der Energieeinsatz geringer ist als bei Glykolyse und Methanolyse (Clarke et al. 1999).

Ammonolyse mit Ammoniak und Aminolyse in Anwesenheit von primären Aminen stellen die am wenigsten erforschten und entwickelten Verfahren da (Clarke et al. 1999). Durch die Umsetzung mit zum Beispiel Methylamin, Ethylamin oder Ethanolamin entstehen Diamide der Terephthalsäure, die mannigfaltig als Grundstoff für Additive, in der Pharmazie sowie für Textilfarben dienen können. Dennoch gibt es keine kommerziellen Bestrebungen zum PETRecycling auf diesem Weg, da eine vollständige Umsetzung von PET schwierig ist. Weiters ist $\mathrm{zu}$ beachten, dass unter sauren und basischen Bedingungen die entstehenden Prozessabwässer durch ihren Gehalt an anorganischen Salzen entsprechend nachbehandelt werden müssen (Clarke et al. 1999; George and Kurian 2014).
Die chemische Depolymerisation bietet somit eine praktikable Möglichkeit zur Verwertung von Kunststoffabfällen zu Sekundärwertstoffen, die keine Qualitätseinbußen gegenüber den primären Produkten aufweisen. Durch die Lösung, Abtrennung von Farb- und Störstoffen und weitere Reinigung vor der erneuten Polymerisation können hohe Reinheiten gewährleistet werden, die auch den bedenkenlosen Einsatz in Kontakt mit Lebensmitteln ermöglichen. Da der Mechanismus jedoch auf einen kleinen Teil der aufkommenden Kunststoffabfallvolumina, die Polykondensationskunststoffe, limitiert ist, bietet sich durch diesen Ansatz jedoch nur begrenztes Potenzial zur Erhöhung der Recyclingquoten. Durch die Selektivität des Lösungsprozesses hinsichtlich der Kunststoffsorten kann solch ein Verfahren allerdings gezielt zur Auftrennung von gemischten Kunststoffabfällen angewandt werden. Dabei gehen die löslichen Bestandteile in die Flüssigphase über und können von den unlöslichen Feststoffen abgetrennt werden, wodurch durch Ausfällung anschließend Reinsubstanzen rückgewonnen werden können.

Sowohl glykolytische als auch methanolytische Verfahren befinden sich bereits in industrieller Anwendung zum PET Recycling und werden zum Beispiel von Unternehmen wie DuPont (Dupont) und Eastman (Eastman 2019) realisiert sowie im Zuge des UnPETProzesses eingesetzt. Das industrielle Interesse von Konzernen wie etwa Coca Cola an neuartigen Verfahren, wie zum Beispiel einer hydrolytischen Depolymerisation mithilfe von Mikrowellen zeigt, dass in diesem Bereich Bedarf für
Recyclingalternativen gegeben ist (gr3n recycling; Coca Cola Company 2019).

Neben Polyethylenterephthalat können auch Polyurethane (PUR) durch chemische Depolymerisation recycelt werden, wobei auch hier Wasser und Glykol als chemisches Lösungsmittel zur Anwendung kommen (Clarke et al. 1999). Bei der Hydrolyse entstehen dabei Polyole und Amine, ein Verfahren, das bereits seit den 1970er-Jahren angewandt wird. Weiters entwickelte die Ford Motor Company eine hydroglykolytische Technologie, die nach eigenen Angaben aus verunreinigten Abfällen Produkte hoher Reinheit generiert (Zia et al. 2007). In der EU läuft seit Jänner 2019 des Weiteren ein neues Forschungsprojekt zur Optimierung der chemischen Depolymersiation zum tertiären PUR-Recycling (CORDIS 2019b).

Auch End-of-life-Polyamide (PA), wie zum Beispiel in aliphatischer Form unter dem Trivialnamen Nylon bekannte Verbindungen, werden durch chemische Depolymerisation, hauptsächlich hydrolytisch, verwertet und dadurch Monomere wie Capralactam rückgewonnen (Clarke et al. 1999). Vor allem auch die Möglichkeit der Auftrennung und Verwertung von Verbundwerkstoffen wie carbon- oder glasfaserverstärktes PA durch Chemolyse sei an dieser Stelle erwähnt (Braun et al. 2001; Chaabani et al. 2017). Im Zuge des Projektes RECAM wurde die Anwendbarkeit der chemischen Depolymerisation auf Teppichfasern untersucht (COMM/RTD 2019a) und die Produkte aus auch recycelten Fischernetzen auf diesem Weg unter dem Namen ECONYL ${ }^{\circledR}$ vertrieben.

\subsection{Thermochemisches Recycling - Pyrolyse}

Polymerketten aus Abfallkunststoffen können auch durch das Verweilen bei Temperatur über $300^{\circ} \mathrm{C}$ in inerter Atmosphäre bei der Pyrolyse zu kürzeren Kohlenwasserstoffbestandteilen zerteilt werden. Diese beim Recycling bewusst induzierte thermische Degradation von Kunststoffen zeichnet sich durch ein breites Produktspektrum aus, welches durch den eingesetzten Ausgangsstoffstoff, die Prozessbedingungen und den Reaktortyp beziehungsweise das grundsätzliche Verfahrensdesign bestimmt wird. Dementsprechend ist auch die Verwendung der Produkte vielseitig und reicht über die Verarbeitung in einer konventionellen Raffinerie hin 
zur Erzeugung von Monomereinheiten für die Polymerisation bis zu Grundchemikalien für die Petrochemie und Treibstoffen. Bei einigen Kunststoffsorten wie z.B. Polystyrol wird auch bei der Pyrolyse von einer Depolymerisation gesprochen, da das Polymer bei gewissen Bedingungen durch die gegenläufige Reaktion der Polymerisation in seine Monomere zerfällt. Bei den meisten Massenkunststoffen wird aber ein Gemisch aus unterschiedlich langen Kohlenwasserstoffketten gewonnen, welches als synthetisches Rohöl oder Pyrolyseöl bekannt ist und durch destillative und veredelnde Schritte in marktfähige Produkte weiterverarbeitet werden kann. Durch weitere Prozessschritte wie für konventionelles Rohöl in zum Beispiel einer Ethylencrackanlage erhält man wieder Monomere zur Herstellung von Kunststoffen. Weiters besteht die Möglichkeit, die teilweise ungesättigten Kohlenwasserstoffe mittels Hydrierung in stabilere Verbindungen für die Anwendung als Treibstoffe oder chemische Grundstoffe überzuführen.

Die thermische Degradation läuft nach einem radikalischen Kettenmechanismus ab, welche detailliert zum Beispiel in Clarke et al. (1999) und Scheirs and Kaminsky (2006) zu finden ist. Welche Produkte entstehen, hängt sowohl von der Art des initialen Kettenbruchs und dem Fortsetzungsmechanismus ab. Man unterscheidet mehrere Startmechanismen, die meist von Fehlerstellen oder weniger starken chemischen Bindungen in der Polymerkette ausgehen. Zufällige, initiale Kettenspaltungen führen zu einem statistisch verteilten Produktspektrum, während bei anderen Kunststoffsorten durch Abspaltung von Kettenenden oder funktionellen Gruppen Monomere entstehen, wie es bei Polystyrol der Fall ist. Bei der Pyrolyse von Polyvinylchlorid spaltet sich aufgrund der geringeren Bindungsstärke $\mathrm{HCl} \mathrm{ab}$, bevor die Hauptkette in kürzere Fragmente zerfällt. Durch die Auftrennung einer Bindung entsteht eine Radikalstelle, welche je nach sterischen Effekten an dieser Kettenposition verweilt oder sich inter- oder intramolekular weiterbewegt und anschließend $\mathrm{zu}$ weiteren Abspaltungsschritten führt. Meist treten aber mehrere dieser Mechanismen simultan auf, wodurch die Beschreibung des Reaktionsmechanismus viele komplexe Teilschritte und Zwischenprodukte umfasst.
Die thermische Zersetzung von Kunststoffen läuft bei hohen Temperaturen ab und die Unterteilung der Pyrolyse erfolgt nach der Prozesstemperatur, wobei zwischen langsamer Pyrolyse bei unter $400^{\circ} \mathrm{C}$, moderater Pyrolyse von 400 bis $600^{\circ} \mathrm{C}$ und Schnellpyrolyse über $600^{\circ} \mathrm{C}$ differenziert wird. Dementsprechend muss die Verweilzeit angepasst werden, welche von Sekunden bis vielen Stunden variieren kann. Die Temperatur beeinflusst nicht nur die notwendige Verweildauer des Kunststoffs bis zum vollständigen Umsatz, sondern auch die Produktausbeuten. Grundsätzlich gilt die Regel, dass je höher die Temperatur im Reaktor ist, desto leichtere Produkte, Gas und Koks entstehen, wobei bei niedrigeren Temperaturen mehr Wachse (Aliphaten mit hohem Molekulargewicht) und schwerere Öle entstehen. Der Prozessdruck wird meist unter atmosphärischen Bedingungen gehalten. Der Druckeinfluss ist bisher nicht vollends geklärt, da mehrere Arbeiten zu unterschiedlichen Erkenntnissen kommen (Murata et al. 2004; Schubert et al. 2019). Allerdings hängt dies auch vom Reaktortyp ab, da zum Beispiel der Druck auch Auswirkung auf den Gasphasenanteil hat und dadurch die Verweilzeit signifikant beeinflusst.

Thermochemische Prozesse zeichnen sich durch die breite Anwendbarkeit der Produkte, vor allem der Flüssigphase aus, die in der bestehenden Infrastruktur einer Erdölraffinerie weiterverarbeitet werden können. Weiters ist nur Energie in Form von Wärme für die endotherme Reaktion notwendig, ansonsten werden keine weiteren Betriebsmittel oder Verbrauchsmaterialien benötigt. Durch die mögliche Verunreinigung der Kunststoffabfälle in Form von Rückständen organischer Natur oder Beimengungen und Verbunden bietet sich die Pyrolyse als attraktiver Recyclingprozess an, da die thermische Zersetzung wesentlich toleranter gegenüber Verunreinigungen und Sortenmischungen als mechanische Recyclingrouten ist. Dennoch können Probleme durch gewisse Kunststoffsorten auftreten. So muss vor allem aus Korrosionsgründen der PVC-Gehalt im Einsatz möglichst gering gehalten werden oder Chlor über einen Entchlorungsschritt als Salzsäure abgeschieden werden. Außerdem können Heteroatome die Produktqualität je nach nachfolgender Verwertung verringern und in Folgeanlagen zu Problemen führen. Die
Einbringung von Sauerstoff, Chlor und Schwefel durch die Einsatzmaterialien kann in geringwertigere Produktspezies münden. Bei der thermochemischen Verarbeitung von Polyethylenterephthalat bildet sich außerdem Terephthalsäure, wodurch auch Potenzial für Anlagenverblockungen durch Ausfällung besteht. Als Einsatzmaterialien für die Kunststoffpyrolyse eignen sich daher vor allem Polyolefine, welche nur aus Wasserstoff- und Kohlenstoffatomen bestehen, somit werden keine organisch gebundenen Fremdatome in den Prozess eingebracht. Zusätzlich lassen sich Polyolefine gut sortieren, da sie im Gegensatz zu vielen anderen Kunststoffen leichter als Wasser sind (Scheirs and Kaminsky 2006; Tukker 2002).

Bei der Pyrolyse muss Energie zugeführt werden, da es sich um einen endothermen Prozess handelt. Der thermische Energieeintrag stellt eine der größten Herausforderungen des thermochemischen Kunststoffrecyclings dar, da Kunststoff sehr niedrige Wärmeleitungskoeffizienten aufweist und die Schmelze sehr zähflüssig ist. Aus diesem Grund verfolgt man unterschiedliche Lösungsansätze, wodurch sich unterschiedliche Anlagenkonzepte und Reaktortypen etabliert haben. Eine einfache und stabile Möglichkeit bieten Drehrohröfen, wie beispielsweise der Berliner Prozess für Kunststoffe aus Elektro- und Elektronikabfällen, wohingegen auch reaktive Extrusionsanlagen in Frage kommen (Tukker 2002). Prozesstechnisch ist es jedoch vorteilhaft, die Oberfläche des Kunststoffs möglichst zu vergrößern, um den Wärmeübergang sowie den Stofftransport $\mathrm{zu}$ verbessern und so den Kunststoff schnell zu pyrolysieren. Dazu werden Wirbelschichtreaktoren verwendet, wie zum Beispiel im Hamburger Verfahren, welches auch katalytisch betrieben werden kann (siehe Abschn. 3.3.). In der chemischen Industrie ist vermehrt der konventionelle Rührkessel in Verwendung, als Beispiel dafür ist das BASFVerfahren anzugeben (Scheirs and Kaminsky 2006; Schubert et al. 2019; Tukker 2002).

Ein aktuelles Beispiel für einen Pyrolyseprozess ist das ReOil-Verfahren (OMV 2018), welches sich gerade im Pilotbetrieb in der Raffinerie Schwechat befindet und auch in Schubert et al. (2019) beschrieben wird. Ein flüssiges, hochsiedendes Raffinerienebenprodukt fungiert hierbei als Lösungsmittel, um die Viskosität und Wärmeleitfähigkeit 


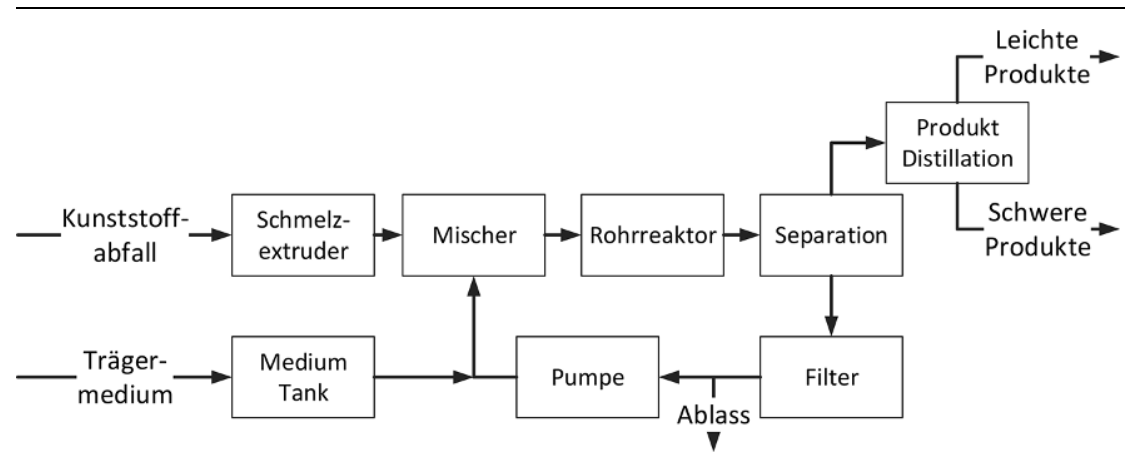

Abb. 5 Schematisches Blockfließbild des ReOil Prozesses. (Nach Schubert et al. 2019)

der Kunststoffschmelze zu verbessern. Der Kunststoff wird in einem Extruder in Schmelzphase übergeführt und so einem Trägermediumkreislauf beigemengt (siehe Abb. 5). Diese Mischung wird in einem Rohrreaktor auf rund $400{ }^{\circ} \mathrm{C}$ erhitzt und der Kunststoff in kurzkettige Kohlenwasserstoffe gespalten, welche anschließend destillativ entfernt werden. Schwere Produktanteile, unkonvertierter Kunststoff und das Trägermedium verbleiben im Kreislauf und werden mit einer Pumpe erneut zur Mischstelle geführt. Die Hauptprodukte sind flüssige Kohlenwasserstoffe unterschiedlicher Siedecharakteristik, welche als synthetisches Rohöl direkt in der Raffinerie weiterverarbeitet werden. Es entstehen auch geringe Anteile an Nebenprodukten wie Gas und Koks (Schubert et al. 2019).

Neben dem ReOil-Verfahren der OMV in Österreich gibt es aktuell hohe Forschungs- und Entwicklungsaktivität im Bereich des thermochemischen Kunststoffrecyclings. So zeigen sich auch bei Neste (Neste 2018), Petrogas (Petrogas 2019) mit der BlueAlp Technology (BluAlp 2019) oder ein NASASpin-Off namens VADXX Energy (Vadxx 2019) andere große Unternehmen, vor allem mit petrochemischem Hintergrund, jüngst interessiert an diesem Ansatz.

\subsection{Thermokatalytisches Recycling}

Eine weitere Möglichkeit, Kunststoffabfälle in kürzere Kohlenwasserstoffe $\mathrm{zu}$ Recyclingzwecken zu konvertieren und Polymerketten zu brechen, ist ähnlich der Pyrolyse - die thermische Spaltung in der Anwesenheit eines katalytisch wirkenden Stoffes. Die thermokatalytischen Prozesse werden in heterogene und homogene Systeme eingeteilt. Heterogene Katalysatoren sind Feststoffe, welche beispielsweise in einem Festbett- oder Wirbelschichtreaktor verwendet werden können. Vielverwendete Katalysatoren sind zum Beispiel Zeolithe, es werden aber auch andere Stoffe wie Metalloxide, Reinmetalle oder metallbeschichtete Kohle eingesetzt. Homogene Katalysatoren sind weniger weit verbreitet und basieren meist auf ionischen Flüssigkeiten. Im Gegensatz zur rein thermischen Pyrolyse hat die katalytische Umsetzung mehrere Vorteile: Die Aktivierungsenergie der Crackingreaktionen wird durch die Anwesenheit des Katalysators verringert, dadurch kann die Temperatur niedriger gehalten werden. Bei gleichbleibenden Bedingungen wird die Reaktion durch den Katalysator beschleunigt. Dadurch ergeben sich Energieeinsparungen und kleinere Reaktoren, was wirtschaftlich vorteilhaft sein kann. Der typische Temperaturbereich thermokatalytischer Prozesse liegt zwischen 300 und $900^{\circ} \mathrm{C}$, je nach eingesetztem Reaktortyp, Druck und gewünschten Produkten. Dementsprechend muss die Verweilzeit der Reaktionstemperatur angepasst werden, sodass diese von Millisekunden bis Stunden betragen kann. Neben den Verfahrensbedingungen, die bei der Pyrolyse als Stellschrauben für die Produkte dienen, ist der Katalysator ein weiterer Prozessparameter, durch den das Produktspektrum beeinflusst werden kann. Im Vergleich zur rein thermischen Degradation entstehen mehr verzweigte, ringförmige und aromatische Verbindungen, die je nach Verwertung einen höheren Wert darstellen. Bei der Einflussnahme auf Ausbeute und Spektrum spielt nicht nur das Katalysatormaterial und dessen Selektivität eine Rolle, auch die Oberfläche bzw. Porenstruktur haben große Auswirkungen. Die Verwendung eines Katalysators setzt jedoch auf der anderen Seite aber auch ein höhere Qualität des verarbeitbaren Einsatzes voraus. Durch spezielle Kunststoffsorten und Verunreinigungen können Heteroatome wie Stickstoff oder Schwefel in den Prozess gelangen, welche als Katalysatorgifte fungieren können und den Umsatz durch Katalysatorinaktivierung mindern. Des Weiteren können auch andere Störstoffe, wie mineralische Füllstoffe oder sich bildender Koks, den Katalysator durch Ablagerungen oder chemische Reaktionen deaktivieren. Dies kann durch eine geeignete Regeneration mit zusätzlichen Aggregaten rückgängig gemacht werden. Nicht zuletzt muss auch darauf geachtet werden, dass Katalysatorrückstände gänzlich aus dem Produkt entfernt werden, was zusätzlichen apparativen Aufwand bedeutet und die Gefahr erhöht, vor allem feinen Katalysatorabrieb im Produkt wiederzufinden (Scheirs and Kaminsky 2006).

Ein Beispiel für ein industrielles, thermokatalytisches Recyclingverfahren ist der Thermofuel-Prozess, in dem die Reaktion in einem Konverterturm stattfindet, welcher nickellegierte Platten als katalytische Einbauten besitzt. Das bereits unter thermochemischem Recycling vorgestellte Hamburger Verfahren (siehe Abb. 6) verwendete Quarzsand, aber auch katalytisch aktive Materialien wie Zeolithe, um mit einem von unten kommenden Stickstoff- oder anderen Inertgasstrom ein Wirbelbett $\mathrm{zu}$ bilden. Der Kunststoff wird über eine Schnecke in den Reaktionsraum der Wirbelschicht eingebracht, welche bei über $400^{\circ} \mathrm{C}$ betrieben wird. Je nach Art des Kunststoffs und Reaktionstemperatur werden dabei aus dem Altkunststoff Monomere wie Styrol oder Methylmethacrylat, Wachse oder Aromaten wie Xylol, Benzol und Toluol gewonnen. Wegen Problemen bei der Maßstabsvergrößerung der Wirbelschicht wurden die Projekte beendet (Scheirs and Kaminsky 2006).

Der Amoco-Prozess ist ebenfalls ein Beispiel für die Verwendung von Zeolith-Katalysatoren, wie auch der FujiRecycling-Prozess, welcher eine katalytische Entchlorung bei $300^{\circ} \mathrm{C}$ als Besonderheit aufweist, um auch PVC störungsfrei einsetzen zu können (Scheirs and Kaminsky 2006).

Der Alphakat NanoFuel Process ist ein thermokatalytischer Prozess basierend auf einem ionentauschfähigen Natrium-Zeolith-Katalysator, der die Fähigkeit besitzt, Halogene zu bin- 


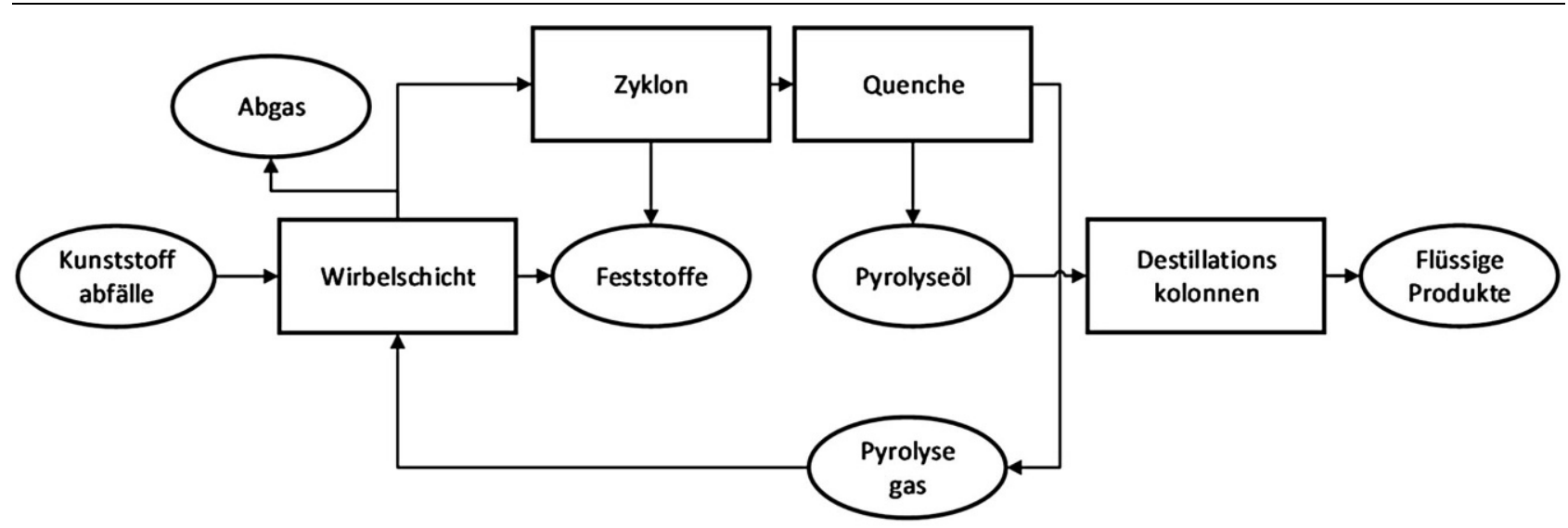

Abb. 6 Schematisches Blockfließbild des Hamburger Verfahrens. (Nach Scheirs and Kaminsky 2006)

den, wodurch auch PVC und andere halogenhaltige Einsatzmaterialien verarbeitet werden können. Der Prozess generiert bis $\mathrm{zu} 95 \%$ Flüssigprodukte, bis zu $5 \%$ Gas und unter einem Prozent feste Rückstände. Der Kunststoffschmelze wird ein schweres, heizölähnliches Öl zugegeben, was auch die thermische Leitfähigkeit der Kunststoffschmelze verbessert. Die Konversion des Kunststoffs findet in Anwesenheit des Katalysators in einem Rührkessel statt, die Hauptprodukte verdampfen und werden am Kopf des Rührkessels gesammelt und destilliert. Der Katalysator und feste Rückstände sammeln sich unten und werden mit einer Förderschnecke abtransportiert. Der Katalysatorverbrauch liegt bei $1,5 \%$ der flüssigen Produktausbeute. Der Rührkesselreaktor wird nahezu drucklos bei 270 bis $390^{\circ} \mathrm{C}$ betrieben, wodurch Koksbildung minimiert wird. Diese Art von katalytischen Crackanlagen wurde in der Größenordnung von 500 Litern pro Stunde in Mexiko, Japan, Korea und Deutschland errichtet (Scheirs and Kaminsky 2006).

Aktuelle Entwicklungen weiterer Verfahren sind in einem Bericht für das American Chemistry Council nachzulesen (4R Sustainability Inc. 2011; OCEAN RECOVERY ALLIANCE 2015). Hierbei sind als katalytische Verfahren die PARC-Technologie (Plastic Advanced Recycling Corp. 2019), die TACTechnologie (englisch thermal anaerobic conversion) von Plastic Energy oder der Cat-HTR (katalytischer Hydrothermal-Reaktor), welcher von den Unternehmen Licella und ReNewELP verwendet wird (Licella 2019b; Plastic Energy 2019; ReNew ELP 2019), zu nennen.

\subsection{Hydrocracking}

Ähnlich wie das thermochemische und katalytische Recycling ist Hydrocracking eine weitere Möglichkeit, Altkunststoffe durch Konversion in kürzerkettige Kohlenwasserstoffe zu rezyklieren. Wie in den zuvor genannten Prozessen werden die Kunststoffketten erhöhten Temperaturen ausgesetzt, um Bindungen zu spalten. Dabei wird noch zusätzlich Wasserstoff mit einem Partialdruck von 2 bis $15 \mathrm{MPa}$, oft in Anwesenheit eines biofunktionellen Katalysators, zur Verfügung gestellt. Dadurch wird die Bildung von ungesättigten Verbindungen unterbunden, somit hauptsächlich Alkane und wenig Alkene und Aromaten im Produkt gebildet und dadurch höhere Produktqualitäten und stabilere Verbindungen erreicht. Die Polymerisationsneigung der gesättigten Kohlenwasserstoffe zur erneuten Kunststoffsynthese ist jedoch vermindert. Der bifunktionelle Katalysator erfüllt zwei Aufgaben: einerseits hat er eine Cracking- bzw. Isomerisierungsfunktion und andererseits eine hydrierende bzw. dehydrierende Funktion. Zuerst wird der zu crackende Kohlenwasserstoff beispielsweise zu einem Olefin dehydriert, wobei Wasserstoff frei wird. Darauf folgt eine Isomerisierung zu verzweigten Olefinen. Als letzter Schritt folgt die Hydrierung, wodurch ein gesättigter Kohlenwasserstoff entsteht. Auf ähnlicher Weise können auch zyklische Paraffine und Aromaten entstehen, was die Oktanzahl der leichten Produkte steigert. Die Zersetzungsreaktionen laufen wie beim thermokatalytischen Cracken bei tieferen Temperaturen als bei einer alleinigen Pyrolyse ab. In Munir et al. (2018) werden optimale Bedingungen für einen bifunktionellen Katalysator mit $400^{\circ} \mathrm{C}$, 20 bis 60 bar und eine Verweilzeit von unter $60 \mathrm{~min}$ vorgeschlagen. Der Wärmebedarf ist nicht nur durch die niedrigen Temperaturen beim Hydrocracking vermindert, sondern auch deshalb, weil die Hydrierung exotherm vonstattengeht und die freiwerdende Wärme für die Spaltung der Polymerketten verwendet werden kann. Allerdings muss die Wasserstoffbereitstellung in die energetische Betrachtung miteinbezogen werden. Der Prozess kann auch zweistufig, mit einer vorgeschalteten Pyrolyse und anschließender Hydrierung, ausgeführt sein. Der Vorteil der getrennten Kettenspaltung und Hydrierung ist, dass Heteroatome, Störstoffe und Koks nach der Pyrolyse entfernt werden können und somit der Katalysator im Hydrierschritt geschont werden kann. Ein Nachteil des Hydrocrackings sind die hohen Kosten für den bifunktionellen Katalysator sowie die Kosten für Wasserstoff und der damit einhergehende Mehraufwand, die den Energieeinsparungen durch den geringeren Wärmebedarf und hohen Flüssigproduktausbeuten gegenüberstehen. Des Weiteren muss, wie beim thermokatalytischen Recycling, auf potenzielle Katalysatorgifte im Abfalleinsatz und Deaktivierung durch Ablagerungen geachtet werden (Scheirs and Kaminsky 2006).

Beispiele für kommerzielle Hydrocracking-Verfahren finden sich in der Literatur (Munir et al. 2018; Samperio 2016; Tukker 2002). Das RWE-Verfahren ist ein Beispiel für eine langsame Pyrolyse mit anschließender Hydrierung. Der Hiedrierwerke-Prozess, der Freiberg-Prozess, der Böhlen-Prozess und das ITC-Verfahren bedienen sich einer simultanen Wasserstoffzu- 


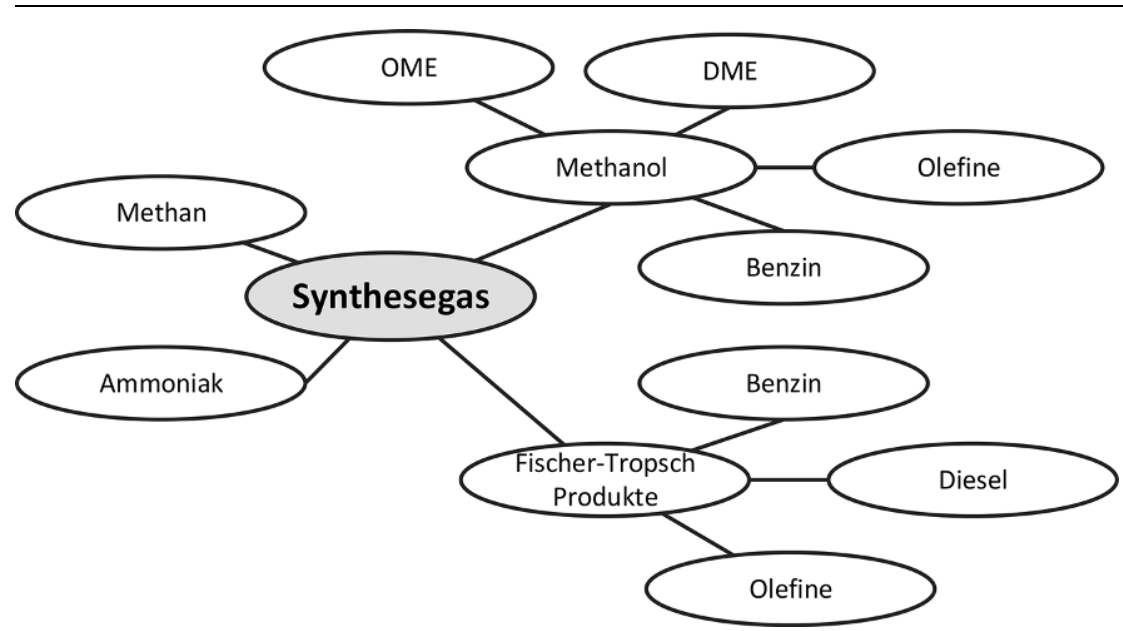

Abb. 7 Synthesegas-Verwertungswege zur Herstellung von chemischen Grundstoffen

fuhr und thermokatalytischen Spaltung für unterschiedliche Kunststoffabfallfraktionen. Ein interessantes, zweistufiges Verfahren stellt der Veba- bzw. VCC-Prozess dar, der ursprünglich für Kohle als Einsatz entwickelt, jedoch kurz vor der Jahrtausendwende für Vakuumrückstand und Kunststoffabfälle adaptiert wurde. Dabei erfolgte die Mischung von Vakuumgasöl mit den Kunststoffabfällen, die in einem Kreislauf bei milden Bedingungen von $400^{\circ} \mathrm{C}$ und atmosphärischem Druck thermisch crackten. Die Produkte wurden anschließend aus dem Kreislauf entfernt und hydriert. 1999 erfolgte die Realisierung dieses Prozesses in einer großtechnischen Anlage, welche aber stillgelegt wurde, da sie zum damaligen Zeitpunkt gegen die energetische Verwertung nicht wettbewerbsfähig war.

\subsection{Gasifizierung}

Unter Gasifizierung oder Vergasung versteht man allgemein die partielle Oxidation von kohlenstoffhaltigen Materialien, die mittels Luft, Sauerstoff, Dampf oder Mischungen daraus, üblicherweise bei Temperaturen zwischen 700 und $1600^{\circ} \mathrm{C}$ und Drücken zwischen 10 und 90 bar vollzogen wird. Diese Technologie wurde ursprünglich zur Umsetzung von Kohle in Synthesegas, ein Gasgemisch aus hauptsächlich Kohlenmonoxid und Wasserstoff, entwickelt, um daraus Energie bereitstellen oder chemische Grundstoffe (siehe Abb. 7) synthetisieren zu können.

Während der Vergasung laufen eine Reihe unterschiedlicher Gleichgewichtsreaktionen ab (siehe Gl. 1 bis
13), wobei sich meist im ersten Schritt der Einsatzstoff thermisch ohne Einwirkung von Sauerstoff oder Wasserdampf zersetzt und erst anschließend teiloxidiert wird.

$$
\begin{aligned}
& \mathrm{C}_{\mathrm{x}} \mathrm{H}_{\mathrm{y}} \leftrightarrow \mathrm{xC}+\mathrm{y} / 2 \mathrm{H}_{2} \\
& \mathrm{C}+1 / 2 \mathrm{O}_{2} \leftrightarrow \mathrm{CO} \\
& \mathrm{CO}+1 / 2 \mathrm{O}_{2} \leftrightarrow \mathrm{CO}_{2} \\
& \mathrm{H}_{2}+1 / 2 \mathrm{O}_{2} \leftrightarrow \mathrm{H}_{2} \mathrm{O} \\
& \mathrm{C}_{\mathrm{x}} \mathrm{H}_{\mathrm{y}}+(\mathrm{x}+\mathrm{y} / 4) \mathrm{O}_{2} \leftrightarrow \mathrm{xCO}_{2}+\mathrm{y} / 2 \mathrm{H}_{2} \mathrm{O} \\
& \mathrm{C}_{\mathrm{x}} \mathrm{H}_{\mathrm{y}}+(\mathrm{x} / 2+\mathrm{y} / 4) \mathrm{O}_{2} \leftrightarrow \mathrm{xCO}+\mathrm{y} / 2 \mathrm{H}_{2} \mathrm{O} \\
& \mathrm{C}+\mathrm{H}_{2} \mathrm{O} \leftrightarrow \mathrm{CO}+\mathrm{H}_{2} \\
& \mathrm{CO}+\mathrm{H}_{2} \mathrm{O} \leftrightarrow \mathrm{CO}_{2}+\mathrm{H}_{2} \\
& \mathrm{C}_{\mathrm{x}} \mathrm{H}_{\mathrm{y}}+\mathrm{xH}_{2} \mathrm{O} \leftrightarrow \mathrm{x} \mathrm{CO}+(\mathrm{y} / 2+\mathrm{x}) \mathrm{H}_{2} \\
& \mathrm{C}+\mathrm{CO}_{2} \leftrightarrow 2 \mathrm{CO} \\
& \mathrm{C}_{\mathrm{x}} \mathrm{H}_{\mathrm{y}}+\mathrm{xCO}_{2} \leftrightarrow 2 \mathrm{x} \mathrm{CO}+\mathrm{y} / 2 \mathrm{H}_{2} \\
& \mathrm{CO}+3 \mathrm{H}_{2} \leftrightarrow \mathrm{CH}_{4}+\mathrm{H}_{2} \mathrm{O} \\
& \mathrm{CO}_{2}+4 \mathrm{H}_{2} \leftrightarrow \mathrm{CH}_{4}+2 \mathrm{H}_{2} \mathrm{O}
\end{aligned}
$$

Die freiwerdende Energie der exothermen Reaktionen wie der Reaktion von Kohlenstoff oder Wasserstoff mit Sauerstoff (Reaktionen (1) bis (6)) steht für endotherme Teilreaktionen, zum Beispiel die Boudouardreaktion (10), Methanisierung (12) und (13) sowie die Wassergas-Shift-Reaktion (8) zur Verfügung. Je nach Einsatzstoff, Vergasungsmittel und -technologie setzt sich das Produktgas aus veränderlichen Anteilen $\mathrm{CO}, \mathrm{H}_{2}$ aber auch $\mathrm{CH}_{4}$ und höheren Kohlenwasserstoffen sowie einer Reihe von Verbindungen mit Heteroatomen zusammen (Clarke et al. 1999).

Altkunststoffe und kunststoffhaltige Abfallfraktionen eignen sich ebenfalls für die Vergasung, und wenn das erzeugte Synthesegas zur Erzeugung von chemischen Grundstoffen oder Aus- gangsmaterialen für Synthesen eingesetzt wird, ist diese Technologie dem rohstofflichen Recycling zuzurechnen. So können zum Beispiel $\mathrm{CO}$ und $\mathrm{CO}_{2}$ katalytisch hydrogeniert werden, um Methanol zu gewinnen, das Synthesegas zur Ammoniakherstellung genutzt oder über Fischer-Tropsch-Synthese Kohlenwasserstoffe generiert werden. Die Oxosynthese bietet durch die Hydroformylierung von Olefinen weiters die Möglichkeit, Oxygenate zu synthetisieren. Dampfvergasung liefert aufgrund des höheren Wasserstoffgehalts für etwaige chemische Synthesen hierfür ein günstigeres Ausgangsprodukt. Da für all diese Prozesse der Einsatz von Katalysatoren erforderlich ist, die empfindlich auf Verunreinigungen im Eduktgas reagieren, kommt der Gasreinigung nach der Gasifizierung große Bedeutung zu. Im Falle von Kunststoffen als Einsatzstoff sind vor allem chlorund stickstoffhaltige Komponenten sowie Teere und partikuläre Verunreinigungen zu entfernen (Clarke et al. 1999; Lopez et al. 2018).

Ein großer Vorteil der Gasifizierung von Kunststoffabfällen sind die geringen Reinheitsanforderungen an das Einsatzmaterial, wodurch der Sortieraufwand auch gegenüber der pyrolytischen Wiederverwertung erheblich verringert werden kann (Lopez et al. 2018). Dabei ist zu erwähnen, dass nicht nur eine sortengemischte Aufgabe von Altkunststoffen, sondern auch eine gleichzeitige Verarbeitung mit anderen, festen Abfallstoffen, zum Beispiel in Form von biogenen Reststoffen in gemischten Siedlungsabfällen möglich ist, worauf sich auch der Hauptteil der Forschungs- und Entwicklungsaktivitäten konzentriert (Clarke et al. 1999). Demgegenüber steht, dass durch die partielle Oxidation der erhöhte Sauerstoffgehalt des Synthesegases dessen Energiegehalt mindert und daraus auch die Synthese von sauerstofffreien Verbindungen nicht mehr möglich ist. So können theoretisch nur über Umwege und erhöhte Energieeinbringung wieder gleichwertige Produkte wie Kunststoffe erzeugt werden. Um ein für die Synthese günstigeres H/C-Verhältnis durch Dampfvergasung zu erreichen, werden jedoch bei der Kunststoffvergasung ein erhöhter Energiebedarf zur Umsetzung mit Wasserdampf und höhere Teergehalte im Produktgas im Vergleich zu Biomasse und anderen Einsatzstoffen in Kauf genommen. Durch den Einsatz von gemischten Fraktionen 


\begin{tabular}{|c|c|c|}
\hline & Vorteile & Nachteile \\
\hline Solvolyse & $\begin{array}{l}\text { Sehr selektiv gegenüber speziellen Polymeren } \\
\text { Monomere direkt zur erneuten Kunststoffsynthese }\end{array}$ & Lösungsmittelabtrennung und -handhabung aufwändig \\
\hline Pyrolyse & $\begin{array}{l}\text { Breite Anwendbarkeit der Produkte } \\
\text { Wenig empfindlich gegenüber Heteroatomen (kein Kataly- } \\
\text { sator) } \\
\text { Hoher Energiegehalt der Produkte } \\
\text { Einfaches Anlagendesign }\end{array}$ & $\begin{array}{l}\text { Wenig selektiv } \rightarrow \text { breites Produktspektrum } \\
\text { Produktverarbeitung aufwendig } \\
\text { Chemisch gebundene Heteroatome je nach Anwendung problematisch } \\
\text { Energieeinbringung für endotherme Reaktionen } \\
\text { Verkokungsneigung }\end{array}$ \\
\hline $\begin{array}{l}\text { Thermokatalytisches } \\
\text { Cracken }\end{array}$ & $\begin{array}{l}\text { Breite Anwendbarkeit der Produkte } \\
\text { Produktspektrum teilweise steuerbar } \\
\text { Geringerer Wärmebedarf durch Katalysator }\end{array}$ & $\begin{array}{l}\text { Katalysator empfindlich gegenüber speziellen Heteroatomen } \\
\text { Katalysatorabtrennung prozesstechnisch aufwendig } \\
\text { Verkokungsneigung }\end{array}$ \\
\hline Hydrocracking & $\begin{array}{l}\text { Geringere Koksbildung durch Wasserstoff } \\
\text { Stabilere, gesättigte Produkte } \\
\text { Geringerer thermischer Energiebedarf durch exotherme } \\
\text { Hydrierung }\end{array}$ & $\begin{array}{l}\text { Energiebedarf zur Wasserstoffbereitstellung } \\
\text { Komplexe Anlagentechnik }\end{array}$ \\
\hline Gasifizierung & $\begin{array}{l}\text { Wenig Einsatzaufbereitung notwendig } \\
\text { Vielfältig einsetzbares Produktgas }\end{array}$ & $\begin{array}{l}\text { Niedermolekulare Produkte } \\
\text { Oxygenierung und dadurch reduzierter Wert der Produkte }\end{array}$ \\
\hline
\end{tabular}

sowie Katalysatoren zur Teerspaltung kann dem jedoch entgegengewirkt werden (Wilk and Hofbauer 2013).

Eine Möglichkeit zur Reduktion von Störstoffen ist der Einsatz von Kalk zur Bindung von Chlor und Schwefel. Dieser Ansatz wird zum Beispiel durch einen Kalkkreislauf in einem Schachtreaktor im Verfahren der Firma ecoloop angewandt, die eine 32-MW-Anlage in Traunstein betreibt, um Synthesegas aus Kunststoffabfällen und anderen Reststoffen zu gewinnen (ecoloop $\mathrm{GmbH}$ 2019). In der Technologie von Texaco zur Gasifizierung von gemischten Kunststoffen, die in den 1990erJahren in einer Pilotanlage für $10 \mathrm{t} / \mathrm{d}$ getestet wurde, erfolgt die Vergasung in einem zweistufigen Verfahren, wobei im ersten Verflüssigungsschritt chlorhaltiges Gas ausgetrieben wird und abgeschieden werden kann. Der entstehende, flüssige Rückstand wird anschließend zur Produktgasgewinnung vergast und enthält somit wesentlich geringere Störstoffgehalte. Eine kommerzielle Anlage war ursprünglich in Dänemark angedacht, wurde jedoch aufgrund von fehlenden Übereinkommen nie realisiert (Tukker 2002). Weiters betreibt das kanadische Unternehmen Enerkem eine industrielle Vergasungsanlage für gemischte, feste Abfälle in der Bioraffinerie in Alberta, Kanada. In einer blasenbildenden Wirbelschicht wird Synthesegas aus sortierten, behandelten Siedlungsabfällen erzeugt, was aufgrund der Zusammensetzung $\mathrm{zu}$ geringeren Teergehalten führt, und anschließend katalytisch zu Ethanol und Methanol sowie weiteren Folgeprodukten umgesetzt (Enerkem).
3.6 Chancen und Herausforderungen der unterschiedlichen Verfahren bei der großtechnischen Realisierung

Wie in den vorhergegangenen Kapiteln dargestellt, wird eine Fülle an Ansätzen zum chemischen Recycling von Abfallpolymeren verfolgt und erforscht. Bedingt durch die Vielzahl an unterschiedlichen chemischen Strukturen, Eigenschaften sowie der Mannigfaltigkeit der Abfallströme eignen sich unterschiedliche Verfahren verschieden gut für explizite Anwendungen. Tab. 3 stellt die Vor- und Nachteile all dieser möglichen Prozesspfade gegenüber, wobei ersichtlich wird, dass die Wahl des am besten geeigneten chemischen Recyclingkonzepts je nach konkreter Fragestellung und Zielsetzung sehr unterschiedlich ausfällt.

Die Breite der Möglichkeiten zum chemischen Recycling spiegelt sich auch in den Kommerzialisierungsansätzen wider, die bereits im letzten Drittel des letzten Jahrhunderts unternommen, jedoch meist aufgrund mangelnder Wirtschaftlichkeit wieder eingestellt wurden. Die ökonomische Konkurrenzfähigkeit der Verfahren zur werkstofflichen Wiederverwertung mit fossilen Alternativen sowie mit anderen Verwertungswegen (z.B. energetische Verwertung) wurde durch folgende prozesstechnische, einsatzlogistische sowie produktseitige Hürden in der Vergangenheit erschwert:

- Fehlende Einsatzverfügbarkeit in geforderter Qualität.

- Variabilität der Einsatzqualität.

- Fehlende Zusicherung der notwendigen Gate Fee.

- Aufwendige Vorbehandlung und Sortierung.
- Handhabung Kunststoffschmelze.

- Verminderung des Wärmeeintrages durch Verkokung und Anlagerungen an den Wärmeübertragerflächen.

- Verblockungen durch Wachse und hochmolekulare Zwischenprodukte.

- Verblockungen durch Calciumchlorid.

- Aufwendige Reinigung durch Verkokungen und Verblockungen.

- Produkte minderer Qualität durch breites Produktspektrum und instabile, chemische Verbindungen.

- Produkte minderer Qualität durch Verunreinigungen und Fremdatome.

- Geringere Ausbeuten.

- Anlagentechnische Schwierigkeiten bei der Maßstabsvergrößerung.

In der jüngsten Vergangenheit sind das Interesse und die Aktivitäten im Bereich des chemischen Recyclings von industrieller Seite erneut gestiegen, was vor allem durch den gesellschaftlichen Druck zur nachhaltigen Lösung der Verwertungsthematik für Kunststoffabfälle, aber auch der zunehmenden Wirtschaftlichkeit gegenüber fossilen Rohstoffen geschuldet ist. Dies zeigt sich auch in Tab. 4, in der weitere, aktuelle kommerzielle Ansätze zusammengefasst sind.

\section{Zusammenfassung}

Um die Möglichkeiten des Recyclings weiter auszubauen und auch Abfallströme mit Kunststoffmischungen bzw. allgemein heterogener Zusammensetzung mit hohem Kunststoffanteil für die stoffliche Verwertung zu erfassen, bietet das chemische Recycling eine breite Palette an Möglichkeiten, chemische Bausteine aus Altkunststoffen rückzu- 


\begin{tabular}{|c|c|c|c|c|c|c|}
\hline Prozess & Unternehmen & Verfahren & Einsatz & Status & $\begin{array}{l}\text { Kapazität } \\
\text { [t/a] }\end{array}$ & Quelle \\
\hline $\begin{array}{l}\text { BlueAlp } \\
\text { nology Tech- }\end{array}$ & BlueAlp & Pyrolyse & Polyolefine & - & 20.000 & $\begin{array}{l}\text { (BluAlp 2019; Petro- } \\
\text { gas 2019) }\end{array}$ \\
\hline Re0il & OMV & Pyrolyse & Polyolefine & Pilotanlage & 800 & (OMV 2018) \\
\hline Cat-HTRTM & Licella Holdings Ltd & $\begin{array}{l}\text { Katalytische hydrothermale } \\
\text { Umsetzung (Hydrocracking } \\
\text { mit Wasser) }\end{array}$ & $\begin{array}{l}\text { Biomasse, Kunststoff- } \\
\text { abfälle, Altöl }\end{array}$ & $\begin{array}{l}\text { Kommerzielle } \\
\text { Anlagen }\end{array}$ & - & (Licella 2019a) \\
\hline $\begin{array}{l}\text { Thermal Anaero- } \\
\text { bic Conversion } \\
\text { (TAC) }\end{array}$ & Plastic Energy & Pyrolyse & Kunststoffabfälle & - & $\begin{array}{l}200.000 \\
\text { ab } 2020\end{array}$ & (Plastic Energy) \\
\hline Vadxx Prozess & Vadxx & Pyrolyse & Kunststoffabfälle & - & 20.000 & (Vadxx 2019) \\
\hline Agilyx Prozess & Agilyx & PS Depolymerisierung & Polystyrol & - & $\sim 3500$ & (Home: Agilyx 2019) \\
\hline $\begin{array}{l}\text { Cassandra Oil's } \\
\text { technology }\end{array}$ & Cassandra Oil & Katalytisches Cracking & $\begin{array}{l}\text { Reifen, Kunststoffab- } \\
\text { fall ohne PVC }\end{array}$ & $\begin{array}{l}\text { Kommerzielles } \\
\text { Patent }\end{array}$ & - & (Cassandra Oil 2019) \\
\hline $\begin{array}{l}\text { Clariter's chemi- } \\
\text { cal process }\end{array}$ & Clariter & Pyrolyse & PE, PP, PS & $\begin{array}{l}\text { Industrielle } \\
\text { Anlage }\end{array}$ & 10.000 & $\begin{array}{l}\text { (Clariter-global } \\
\text { clean-tech group } \\
\text { 2019) }\end{array}$ \\
\hline $\mathrm{IH}^{2}$ & $\begin{array}{l}\text { Illinois-based Gas Techno- } \\
\text { logy Institute/CRI Catalyst/ } \\
\text { Shell }\end{array}$ & Hydrocracking & $\begin{array}{l}\text { Siedlungsabfall, } \\
\text { Biomasse, Kunststoff- } \\
\text { abfälle }\end{array}$ & $\begin{array}{l}\text { Industrielle } \\
\text { Anlage }\end{array}$ & 700.000 & $\begin{array}{l}\text { (IH² Technology; } \\
\text { Shell 2019a) }\end{array}$ \\
\hline DeFuel & Demont & Katalytisches Cracking & Kunststoffabfälle & - & - & (Demont 2019) \\
\hline Enerkem Prozess & Enerkem & Vergasung & $\begin{array}{l}\text { Biomasse, Siedlungs- } \\
\text { abfall, Kunststoff }\end{array}$ & $\begin{array}{l}\text { Kommerzielle } \\
\text { Anlagen }\end{array}$ & 360.000 & (Enerkem 2019) \\
\hline Fulcrum Prozess & Fulcrum & Vergasung & $\begin{array}{l}\text { Fester Siedlungsab- } \\
\text { fall }\end{array}$ & Demoanlage & 175.000 & $\begin{array}{l}\text { (Fulcrum BioEnergy } \\
\text { 2019) }\end{array}$ \\
\hline $\begin{array}{l}\text { GreenMantra } \\
\text { Technologies }\end{array}$ & GreenMantra & Katalytisches Cracking & Polyolefine & - & - & $\begin{array}{l}\text { (Home - GreenMan- } \\
\text { tra 2019) }\end{array}$ \\
\hline $\begin{array}{l}\text { Handerek Pro- } \\
\text { zess }\end{array}$ & Handerek & Hydrocracking & Polyolfefine, PS & - & - & $\begin{array}{l}\text { (Handerek Technolo- } \\
\text { gies 2019) }\end{array}$ \\
\hline $\begin{array}{l}\text { Mehrere Prozes- } \\
\text { se }\end{array}$ & Klean Industries & Pyrolyse und Vergasung & Kunststoffabfälle & $\begin{array}{l}\text { Kommerzielle } \\
\text { Anlagen }\end{array}$ & - & $\begin{array}{l}\text { (Klean Industries } \\
\text { 2019) }\end{array}$ \\
\hline $\begin{array}{l}\text { Quantafuel } \\
\text { Prozess }\end{array}$ & Quantafuel & Katalytisches Cracking & Kunststoffabfälle & $\begin{array}{l}\text { Kommerzielle } \\
\text { Anlag }\end{array}$ & 18.000 & (QuantaFuel 2019) \\
\hline RT7000 & Recycling Technologies & Pyrolyse & Kunststoffabfälle & - & 9000 & $\begin{array}{l}\text { (The RT7000 I Recy- } \\
\text { cling Technologies } \\
\text { 2018) }\end{array}$ \\
\hline LuxCR $^{\mathrm{TM}}$ & DuPont Teijin Films & Solvolyse & PET & - & - & (DuPont 2019) \\
\hline Solvolyse & EASTMAN & Methanolyse & Polyester & $\begin{array}{l}\text { Kommerzielle } \\
\text { Anlage geplant }\end{array}$ & - & (Eastman 2019) \\
\hline $\begin{array}{l}\text { DEMETO Techno- } \\
\text { logie }\end{array}$ & gr3n & $\begin{array}{l}\text { Depolymerisation mit Mikro- } \\
\text { wellen }\end{array}$ & PET & $\begin{array}{l}\text { Industrielle } \\
\text { Anlage } 2021 \\
\text { geplant }\end{array}$ & - & $\begin{array}{l}\text { (gr3n recycling } \\
\text { 2019) }\end{array}$ \\
\hline
\end{tabular}

gewinnen. Vor allem Verpackungsabfälle, die sich aus einem großen Anteil an Polyolefinen zusammensetzen und außerdem aufgrund der kurzen Produktlebensdauer in großen Mengen anfallen, eignen sich in gemischten Abfallfraktionen als Einsatzstoff sehr gut, wodurch rohstoffliches Recycling zur Erreichung der Recyclingziele beitragen kann.

Verfahrenstechnisch ergeben sich durch chemische Wiederverwertung einige Vorteile, vor allem begründet durch den geringeren Reinheitsanspruch des chemischen Recyclings im Vergleich zum mechanischen. Der Sortieraufwand für gemischte Abfälle und Verpackungsabfälle kann redu- ziert werden, wobei je nach Verfahren unterschiedliche Toleranzen möglich sind. Bei der Vergasung zum Beispiel können Siedlungsabfälle ohne jegliche Vorbehandlung umgesetzt werden und auch solvolytische Verfahren ermöglichen stark heterogene Einsatzstoffe. Beide Ansätze ermöglichen zudem die Verwertung von Verbundwerkstoffen, was bedingt auch bei den anderen, hier vorgestellten Verfahren möglich ist. Bei thermochemischen Prozessen bestimmt die Zusammensetzung zunehmend die Qualität der Produkte, und katalytische Prozesse erfordern die Evaluierung von potenziellen Katalysatorgiften und etwaig notwendige
Sortierschritte zur Eliminierung katalysatorschädlicher Fraktionen.

Die entstehenden Produkte, je nach gewähltem chemischen Recyclingverfahren und eingesetzten Kunststoffsorten, Synthesegas, Monomereinheiten oder flüssige Kohlenwasserstoffgemische, bedienen einen breiten Markt und können in der bestehenden Infrastruktur weiterverarbeitet werden. So kann zum Beispiel das bei der Pyrolyse entstehende sogenannte Syncrude in konventionellen Erdölraffinerien weiterverarbeitet und ohne Qualitätsverluste über Ethylencracking-Anlagen wieder in Grundstoffe zur Kunststoffproduktion überführt werden. Dadurch, dass es sich bei den entstehenden Sub- 
stanzen um chemische Grundbausteine handelt, bieten sich vielfältige Anwendungsmöglichkeiten wie die Synthese von diversen anderen Chemikalien aus Synthesegas sowie die Bedienung der gesamten Petrochemie. Durch die Rückführung in Monomere und niedermolekulare Verbindungen wird außerdem ermöglicht, Produktqualitäten vergleichbar mit Primärprodukten zu erreichen und somit ein wirkliches Recycling statt eines Downcyclings $\mathrm{zu}$ minderwertigeren Produkten aus Mischfraktionen zu realisieren.

All diesen Vorteilen stehen vor allem anlagen- und prozesstechnische Hürden gegenüber, die aufgrund der Eigenschaften von Polymeren überwunden werden müssen, um technisch und wirtschaftlich sinnvolle Prozesse $\mathrm{zu}$ entwickeln, was sich auch in der Mannigfaltigkeit der chemischen Recyclingkonzepte zeigt. Weiters ist speziell im Fall der Weiterverarbeitung der Produkte in bestehender Infrastruktur, wie zum Beispiel der Raffination von Pyrolyseölen in petrochemischen Industrieanlagen, die rechtliche Lage und Zurechenbarkeit zu Recyclingraten, vor allem im Fall der Weiterverarbeitung in konventionellen petrochemischen Infrastrukturen, zu klären.

Außerdem haben bisherige Versuche der großtechnischen Realisierung gezeigt, dass bei allen Verfahrensvari- anten des chemischen Recyclings Intermediate und keine fertigen Produkte wie beim werkstofflichen Recycling gewonnen werden. Daher ist eine verlässliche und umfassende Anbindung an Infrastruktur zur Weiterverarbeitung unerlässlich, welche schon im Planungsstadium mitbedacht werden muss, um vermarktbare Chemikalien und Materialien wirtschaftlich erzeugen zu können.

Neben den produktbezogenen Herausforderungen spielen die Einsatzverfügbarkeit in entsprechender Qualität und der etwaige Sortier- und Vorbehandlungsaufwand zur Erreichung der geforderten Reinheit eine tragende Rolle bei der erfolgreichen Realisierung des chemischen Recyclings im Industriemaßstab. Für einen störungs- und unterbrechungsfreien Betrieb ist daher die langfristige Versorgung mit geeigneten Abfallfraktionen zu gewährleisten und entsprechende Sortier- und Vorbehandlungsschritte abgestimmt auf die Stoffströme auszulegen.

Nichtsdestotrotz lohnt es sich, zukünftig das breite Spektrum des chemischen Recyclings vermehrt in den Fokus der Aufmerksamkeit zu rücken, um höhere Anteile an vor allem gemischt anfallenden Kunststoffabfällen werkstofflich $\mathrm{zu}$ verwerten und somit die wertstofflichen Senken bei der Verbrennung und Deponierung zu ver- meiden. Das Potenzial des chemischen Recyclings spiegelt sich auch in der aktuellen Aufmerksamkeit großer, vor allem petrochemischer Konzerne wie OMV (OMV 2018), Neste (Neste 2018) und BASF (BASF for the first time makes products with chemically recycled plastics), aber auch kunststoffverarbeitender und Lebensmittelindustrie wider, die vermehrt versuchen, dem Konsumentenwunsch nach nachhaltigen Verwertungskonzepten und Kreislauflösungen durch Forschungs- und Entwicklungsaktivitäten im Bereich des rohstofflichen Recyclings nachzukommen.

Funding Open access funding provided by Montanuniversität Leoben.

Open Access Dieser Artikel wird unter der Creative Commons Namensnennung 4.0 International Lizenz (http:// creativecommons.org/licenses/by/4. $0 /$ deed.de) veröffentlicht, welche die Nutzung, Vervielfältigung, Bearbeitung, Verbreitung und Wiedergabe in jeglichem Medium und Format erlaubt, sofern Sie den/die ursprünglichen $\mathrm{Au}$ tor(en) und die Quelle ordnungsgemäß nennen, einen Link zur Creative Commons Lizenz beifügen und angeben, ob Änderungen vorgenommen wurden.

\section{Literatur}

4R Sustainability Inc. (2011) Conversion technology: A complement to plastic recycling. https://plastics.americanchemistry.com/Plasticsto-Oil/. Accessed 9 September 2019

Agilyx. https://www.agilyx.com/. Accessed 24 September 2019

Bilitewski B, Härdtle G, Marek K (2000) Abfallwirtschaft: Handbuch für Praxis und Lehre, Dritte, neubearbeitete Auflage. Springer Berlin Heidelberg, Berlin, Heidelberg, s.l.

BluAlp (2019) BluAlp. https://www.bluealp.nl/. Braess Qd, Disselhoff R, Guckel C, Illing G (2001) Rohstoffliches Recycling von glasfaserverstärktem Polyamid-6. Chem.-Ing.-Tech. 73:183-190. https://doi.org/10.1002/1522-2640(200103)73:3<183:

CITE183>3.0.CO;2-J

Bundesgesetz über eine nachhaltige Abfallwirtschaft (Abfallwirtschaftsgesetz 2002 - AWG 2002): AWG 2002

Cassandra Oil. http://www.cassandraoil.com/ en/. Accessed 24 September 2019

Chaabani C, Weiss-Hortala E, Soudais Y (2017) Impact of Solvolysis Process on Both Depolymerization Kinetics of Nylon 6 and Recycling Carbon Fibers from Waste Composite. Waste Biomass Valor 8:2853-2865. https://doi.org/10. 1007/s12649-017-9901-5

Clariter-global clean-tech group. http://www. clariter.com/. Accessed 24 September 2019
Clarke JH, Aguado J, Serrano DA (1999) Feedstock recycling of plastic wastes. RSC Clean Technology monographs. Cambridge, LaVergne: The Royal Society of Chemistry, Ingram Publisher Services

Coca Cola Company (2019) Chemical Recycling: Could This Breakthrough Technology Curb Plastic Waste? https://www.coca-colacompany. $\mathrm{com} /$ stories/chemical-recycling-could-thisbreakthrough-technology-curb-plastic-waste. Accessed 15 August 2019

COMM/RTD (2019a) Recycling von Teppichmaterial | Result in Brief | FP4 | CORDIS | European Commission. https://cordis.europa.eu/project/ AIfon/30976/brief/de. Accessed 15 August 2019 CORDIS (2019b) PolyUrethane Recycling towards a Smart Circular Economy | PUReSmart Project | H2020 | CORDIS | European Commission. https://cordis.europa.eu/project/rcn/ 220032/factsheet/en. Accessed 14 August 2019 Demont De Fuel: from plastic to opportunity-DEMONT srl. http://www.demont.it/defuel/?lang=en. Accessed 24 September 2019 DuPont Teijin Films (2019) DuPont Teijin Films makes a significant step forward in chemical recycling with the LuxCR ${ }^{\mathrm{TM}}$ depolymerisation process. http://europe.dupontteijinfilms. com/news/2019/dupont-teijin-films-makes-asignificant-step-forward-in-chemical-recyclingwith-the-luxcr-depolymerisation-process/. Accessed 13 August 2019
Eastman (2019) Eastman offers innovative recycling technology for polyesters. https://www. eastman.com/Company/News_Center/2019/ Pages/Eastman-offers-innovative-recyclingtechnology-for-polyesters.aspx. Accessed 13 August 2019

ecoloop GmbH. https://www.ecoloop.eu/. Accessed 9 August 2019

Enerkem: From waste to Cellulosic ethanol, Biomethanol | Disruptive technology. https:// enerkem.com/. Accessed 9 August 2019

Fulcrum BioEnergy. http://fulcrum-bioenergy. com/. Accessed 24 September 2019

George N, Kurian T (2014) Recent Developments in the Chemical Recycling of Postconsumer Poly(ethylene terephthalate) Waste. Ind Eng. Chem. Res. 53:14185-14198. https://doi. org/10.1021/ie501995m

gr3n recycling: | Long life to plastic bottles http://gr3n-recycling.com/\#tab-id-10. Accessed 15 August 2019

GreenMantra Technologies (2019). Webpräsenz. http://greenmantra.com/. Accessed 24 September 2019

Handerek Technologies. http://handerektechnologies.com/en/. Accessed 24 September 2019

Klean Industries: Pyrolysis Plant Manufacture \& Gasification Equipment Supplier | Turning Tires, Plastics \& Municipal Solid Wastes into Ener- 
gy. http://www.kleanindustries.com/s/Home. asp. Accessed 24 September 2019

Licella (2019a) Cat-HTR ${ }^{\mathrm{TM}}$. https://www.licella. com.au/cat-htr/. Accessed 24 September 2019

Licella (2019b) Webpräsenz. https://www.licella. com.au/. Accessed 11 September 2019

Lopez G, Artetxe M, Amutio M, Alvarez J, Bilbao $J$, Olazar $M$ (2018) Recent advances in the gasification of waste plastics. A critical overview. Renewable and Sustainable Energy Reviews 82:576-596. https://doi.org/10.1016/j. rser.2017.09.032

Martens H, Goldmann D (2016) Recyclingtechnik: Fachbuch für Lehre und Praxis, 2. Auflage. Springer Vieweg, Wiesbaden

Michaeli W, Bittner M, Wolters L (1993) Stoffliches Kunststoff-Recycling: Anlagen, Komponenten, Hersteller. Hanser, München

Munir D, Irfan MF, Usman MR (2018) Hydrocracking of virgin and waste plastics: A detailed review. Renewable and Sustainable Energy Reviews 90:490-515. https://doi.org/10.1016/j.rser. 2018.03.034

Murata K, Sato K, Sakata Y (2004) Effect of pressure on thermal degradation of polyethylene. Journal of Analytical and Applied Pyrolysis 71:569-589. https://doi.org/10.1016/j.jaap.2003. 08.010

Neste (2018) Neste aiming to use waste plastic as a raw material for fuels and plastics. https:// www.neste.com/releases-and-news/circulareconomy/neste-aiming-use-waste-plasticraw-material-fuels-and-plastics. Accessed 11 September 2019

OCEAN RECOVERY ALLIANCE (2015) 2015 Plastics-to-Fuel Project Developer's Guide. https:// www.oceanrecov.org/assets/files/Valuing_Plastic/ 2015-PTF-Project-Developers-Guide.pdf. Accessed 9 September 2019

OMV (2018) ReOil: Aus Kunststoff wieder Öl gewinnen. https://www.omv.com/de/blog/reoilaus-kunststoff- wieder-oel-gewinnen. Accessed 30 September 2019
Pardal F, Tersac G (2006) Comparative reactivity of glycols in PET glycolysis. Polymer Degradation and Stability 91:2567-2578. https://doi. org/10.1016/j.polymdegradstab.2006.05.016 Petrogas (2019) Plastic to Chemicals | Petrogas. https://www.petrogas.nl/process-systems plastic-to-oil/. Accessed 18 September 2019 Plastic Advanced Recycling Corp. P.A.R.C. Technology. http://www.plastic2x.com/parctechnology/. Accessed 9 September 2019

PlasticsEurope AISBL (2018) Plastics - the Facts 2018. https://www.plasticseurope.org/ application/files/6315/4510/9658/Plastics_the_ facts_2018_AF_web.pdf. Accessed 24 September 2019

Plastic Energy. https://plasticenergy.com/. Accessed 11 September 2019

QuantaFuel:-Produces environmentally friendly, clean and cheap synthetic diesel (not biodiesel) from wasted resources. https://quanta fuel.com/. Accessed 24 September 2019 Ragaert K, Delva L, van Geem K (2017) Mechanical and chemical recycling of solid plastic waste. Waste Manag 69:24-58. https://doi.org/ 10.1016/j.wasman.2017.07.044

The RT7000 | Recycling Technologies. https:// recyclingtechnologies.co.uk/technology/thert7000/. Accessed 24 September 2019

ReNew ELP. https://renewelp.co.uk/our-process/. Accessed 11 September 2019

Richtlinie (EU) 2018/852 des Europäischen Parlaments und des Rates vom 30. Mai 2018 zur Änderung der Richtlinie 94/62/EG über Verpackungen und Verpackungsabfälle: 2015/0275 (COD)

Samperio JAS (2016) Alternative catalytic processes for the valorization of plastic wastes to fuels, Universidad del Pais Vaso

Scheirs J, Kaminsky W (2006) Feedstock recycling and pyrolysis of waste plastics: Converting waste plastics into diesel and other fuels. Wiley series in polymer science. J. Wiley \& Sons, Chichester, UK, Hoboken, NJ
Schubert T, Lehner M, Karner T, Hofer W, Lechleitner A (2019) Influence of reaction pressure on co-pyrolysis of LDPE and a heavy petroleum fraction. Fuel Processing Technology 193:204-211. https://doi.org/10.1016/j.fuproc. 2019.05.016

Sheel A, Pant D (2019) Chemical Depolymerization of PET Bottles via Glycolysis. In: Recycling of Polyethylene Terephthalate Bottles. Thomas S, Rane A, Kanny K, Abitha VK, Thomas MG (Eds.) Elsevier, pp 61-84

Shell (2019a) $\mathrm{IH}^{2}$ Technology. https://www.shell. com/business-customers/catalysts-technologies/ licensed-technologies/benefits-of-biofuels/

ih2-technology.html. Accessed 11 September 2019

Tukker A (2002) Plastics waste: Feedstock recycling, chemical recycling and incineration. Rapra review reports, vol 148. Rapra Technology, Shrewsbury

Vadxx (2019) Vadxx-Plastic to EcoFuel. https:// vadxx.com/. Accessed 24 September 2019 Wilk V, Hofbauer H (2013) Conversion of mixed plastic wastes in a dual fluidized bed steam gasifier. Fuel 107:787-799. https://doi.org/10. 1016/j.fuel.2013.01.068

Zia KM, Bhatti HN, Ahmad Bhatti I (2007) Methods for polyurethane and polyurethane composites, recycling and recovery: A review. Reactive and Functional Polymers 67:675-692. https://doi.org/10.1016/j.reactfunctpolym.2007. 05.004

Hinweis des Verlags Der Verlag bleibt in Hinblick auf geografische Zuordnungen und Gebietsbezeichnungen in veröffentlichten Karten und Institutsadressen neutral. 